\title{
Design framework for reliable and environment aware management of smart environment devices
}

\author{
Adja Ndeye Sylla ${ }^{1 *}$, Maxime Louvel ${ }^{1}$ and Eric Rutten ${ }^{2}$
}

\begin{abstract}
A smart environment is equipped with numerous devices (i.e., sensors, actuators) that are possibly distributed over different locations (e.g., rooms of a smart building). These devices are automatically controlled to achieve different objectives related, for instance, to comfort, security and energy savings. Controlling smart environment devices is not an easy task. This is due to: the heterogeneity of devices, the inconsistencies that can result from communication errors or devices failure, and the conflicting decisions including those caused by environment dependencies. This paper proposes a design framework for the reliable and environment aware management of smart environment devices. The framework is based on the combination of the rule based middleware LINC and the automata based language Heptagon/BZR (H/BZR). It consists of: an abstraction layer for the heterogeneity of devices, a transactional execution mechanism to avoid inconsistencies and a controller that, based on a generic model of the environment, makes appropriate decisions and avoids conflicts. A case study with concrete devices, in the field of building automation, is presented to illustrate the framework.
\end{abstract}

Keywords: Smart environments, Reliability, Transactional middleware, Automata language

\section{Introduction}

Smart environments are equipped with numerous devices that are automatically controlled to achieve different objectives. For instance, a window can be opened to cool or ventilate a room. Controlling smart environments devices raises several problems. First, devices are built by different manufacturers and use heterogeneous communication technologies. Second, a device may become unreachable due to a hardware failure or a communication error. In this case, a command sent to this device is not received and the corresponding action is not performed. Assuming that the action has been performed leads to a runtime inconsistency (inconsistency in the rest of the paper). For instance, sending the command close to a door and assuming that it is closed becomes an inconsistency if the door remains opened due to a communication error or a failure. Third, the decisions taken to achieve the objectives may be conflicting or violate other

\footnotetext{
*Correspondence: AdjaNdeye.Sylla@cea.fr

'Université Grenoble Alpes, CEA, LETI, DACLE, LIALP, F-38000 Grenoble, France Full list of author information is available at the end of the article
}

objectives. Conflicts and violations are either explicit or implicit. Implicit conflicts and violations are due to environment dependencies and are not easy to detect. For instance, opening a window to cool a room can raise the noise level (resp. the $\mathrm{CO}_{2}$ concentration). This can violate an objective that limits the room noise level (resp. the $\mathrm{CO}_{2}$ concentration) at a given threshold.

In the literature, several solutions have been proposed for the reliability of smart environments [1-16]. These solutions use different methods (e.g., model checking, pairwise comparison of rules) to prevent from explicit and/or implicit conflicts and objectives violations. However, using these solutions requires to manually program or model the behaviour of the smart environment. Then, the program or the model is verified to detected specific errors (e.g. conflicts). If an error is detected, the program or the model is manually modified and verified again. This can be tedious because developers have to consider all the combinatorial possible cases. Moreover, these solutions do not handle the inconsistencies due to communication errors and hardware failures. 
This paper proposes a design framework for reliable and environment aware management of smart environment devices. The proposed framework allows for

- Declarative management of devices, by specifying the target objectives and not how to reach them;

- Avoidance of both explicit and implicit conflicts;

- Avoidance of inconsistencies that are caused by communication errors and hardware failures.

The framework is based on the combination of a rule based transactional middleware (LINC [17]) and a reactive language (Heptagon/BZR [18]). As shown in Fig. 1, the proposed framework enables the autonomic management of devices through a variant of the MAPE-K loop [19] called MIADIE-K (Monitoring, Interpretation, Analysis, Decision, Interpretation, Execution and Knowledge) and consists of:

- An abstraction layer: To deal with the heterogeneity of smart environment devices;

- A transactional execution mechanism: To prevent from the occurrence of inconsistencies;

- An environment aware controller: To make appropriate decisions and prevent from both explicit and implicit conflicts. The controller relies on a generic model of the environment. The generic aspect of this model allows to use the same controller for other environments that have the same types of devices (e.g., rooms of a building).

To improve environment monitoring, the proposed framework allows developers to design monitoring rules, in LINC, and create soft sensors from physical sensors. A soft sensor aggregates or transforms the data of one or more physical sensors. The framework also allows developers to design rules that perform actions on the environment assuming that these rules do not interact with potential conflicting devices (which must be handled by the environment aware controller).

The paper is structured as follows. Section 2 gives the background material. Then, Section 3 describes the proposed framework. Section 4 presents how devices are managed using the framework. Section 5 illustrates the framework through a case study, with concrete devices, in the field of building automation. Section 6 discusses related work. Finally, Section 7 concludes the paper and presents the future works.

\section{Background}

The proposed framework relies on a transactional middleware and a reactive language that supports the synthesis of controllers. The transactional middleware enables the communication with devices and avoids inconsistencies. The reactive language enables the declarative management of devices while preventing from conflicts and objectives violations. In this paper, the transactional middleware LINC [17] and the reactive language Heptagon/BZR [18] are used.

\subsection{LINC middleware}

LINC [17] is a rule based middleware used to develop and deploy distributed applications. It has been used in several domains such as building automation [20-22] and wireless sensor networks [23-25].

\subsubsection{LINC concepts}

LINC relies on three paradigms:

- Associative Memory [26]: It is implemented as a set of distributed tuple spaces containing resources (tuples of strings). In LINC, tuple spaces are called

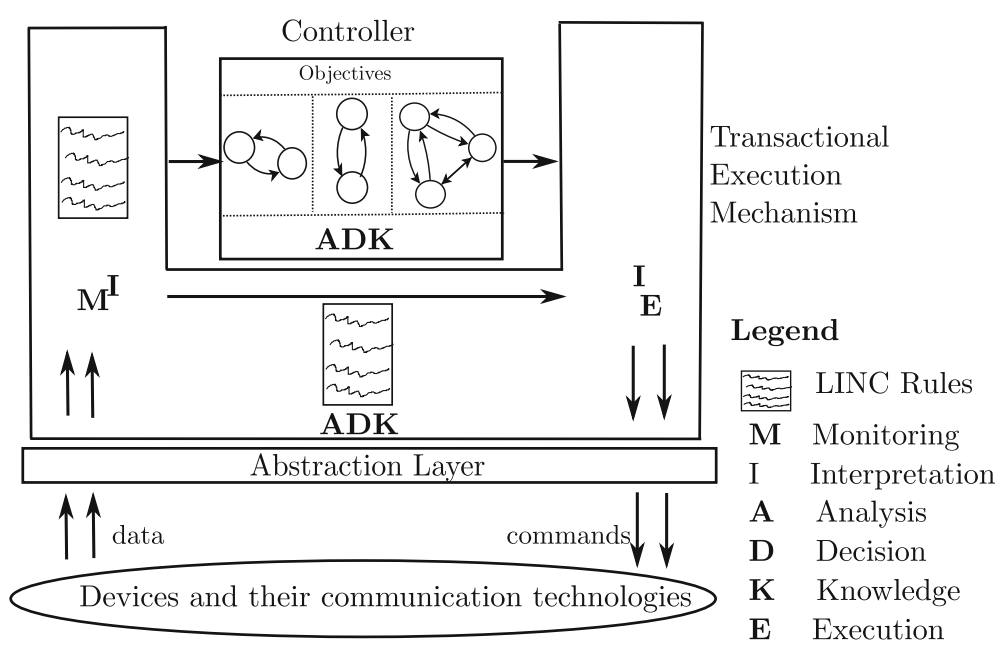

Fig. 1 Devices management through the framework 
bags. They are grouped, according to the application logic, in objects. Resources are used to model the entities of an application and are manipulated using three operations: $r d$, get and put. The $r d$ is used to verify the presence of a resource in a bag. The get is used to remove a resource and the put is used to insert a resource. These operations are used in production rules.

- Production Rules [27]: A production rule consists of two parts: a precondition and a performance. In the precondition, the operation $r d$ is used, with a partially instantiated resource as parameter, to verify specific conditions in the system (e.g., presence detected). If these conditions are true, the performance is triggered. The performance uses the three operations. The $r d$ is used to verify conditions. The get and the put are used to perform actions on the system and update its logical state (i.e., resources stored in LINC bags).

- Distributed Transactions [28]: They are used in the performance part of a rule. A transaction allows to group as one operation the verification of conditions $(\mathrm{rd})$, the realisation of actions (put), and the update of the system logical state (get, put). Thus, the performance part of a rule may abort if, for instance, the verification of a condition through a rd operation is no longer true. The performance part also aborts if a put operation fails because the corresponding action (e.g., switch on a lamp) cannot be performed (e.g., due to a communication error or a hardware failure).

\subsubsection{LINC in the context of smart environments}

LINC provides a framework called PUTUTU [20, 21] that enables the communication with devices and hides their heterogeneity. PUTUTU consists of several LINC objects. As shown in Fig. 2, these objects encapsulate different technologies (e.g., TelosB, LON, Tellstick) and inherit from four generic objects:

- Object_dongles_modules: It is used to manage a dongle or any other equipment plugged in an ethernet or a USB port. The dongle allows to communicate with the devices of a specific technology. It has two bags: Type and Location. Type associates the id of a device to its type. Location associates the id of a device to its location.

- Object_wsan_sensors: It is used to manage sensors. It has one additional bag called Sensors which associates the id of a sensor to its latest measured value, in the format (id, value).

- Object_wsan_actuators: It is used to manage actuators. It has one additional bag called Actuators which is used to send commands to the actuators. The resources of this bag are in the format (id, command, parameters). The insertion of such a resource, using the operation put, actually sends the command to the specified actuator.

- Object_wsan_sensors_actuators: It is used to manage technologies providing both sensors and actuators (e.g. EnOcean). This object is derived from the two previous generic objects and inherits from their bags (e.g., Sensors, Actuators).

\subsubsection{LINC rule example}

Listing 1 presents an example of a LINC rule that switches on the lamp of a room when a presence is detected. This room is equipped with a TelosB presence sensor and an EnOcean lamp actuator.

This rule consists of two parts: a precondition (before the symbol ::) and a performance (after the symbol ::). The precondition consists of a $r d$ on the bag States containing the logical state off and a $r d$ on the bag Sensors of

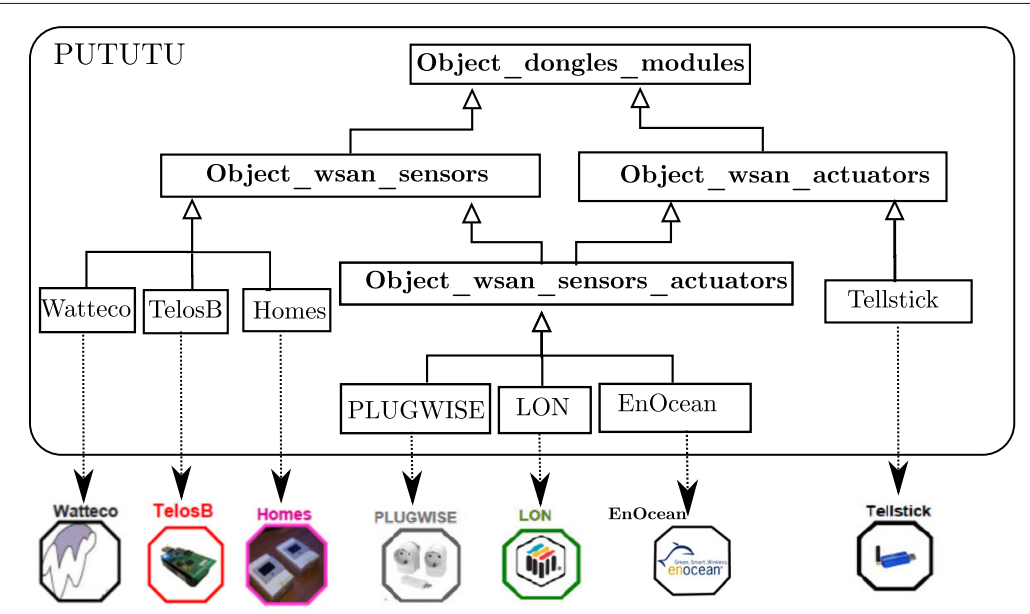

Fig. 2 PUTUTU framework 
the TelosB object to detect a presence. When the lamp is off, the rule waits for a resource indicating a presence in the room. This triggers the performance. The performance consists of two transactions (between \{\} ). The first transaction verifies if the presence is still detected (line 5), sends the command to the lamp (line 6) and updates its logical state (lines 7 and 8). LINC ensures that all the actions are done or none of them. Hence, if the put operation fails on the actuator (e.g., communication error, actuator failure), the lamp stays off in the bag States.

If no error occurs in the first transaction, the second transaction will fail at line 12 (the lamp is now on). If the presence is not detected anymore, both transactions will fail (lines 5 and 11). Finally, if the lamp cannot be switched on (e.g., due to a communication error), the second transaction will send a SMS to the maintenance team to inform them of the problem.

Executing this rule in another room simply requires replacing the PUTUTU objects (i.e., TelosB and EnOcean) and the ids (i.e., t_pr_1, e_l_1), respectively, by the communication technologies and the ids of the room presence sensor and lamp actuator.

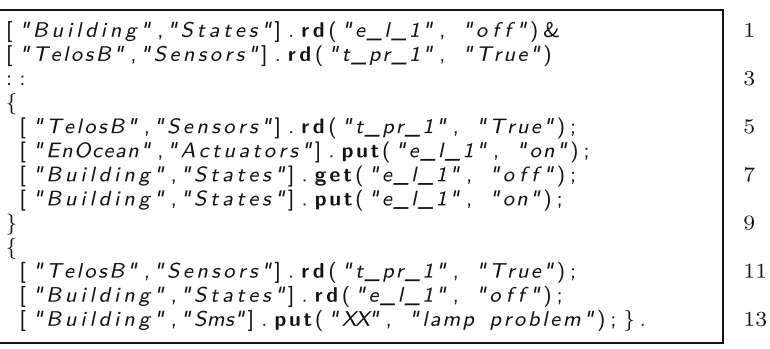

Listing 1 LINC rule example

\subsection{Heptagon/BZR language}

Heptagon/BZR or H/BZR [18] is a language used to build reactive systems, by means of automata and equations. It enables model checking to verify properties (e.g., absence of objectives violations) and especially the synthesis of controllers to enforce properties.

\subsubsection{Design of a H/BZR program}

A H/BZR program is designed as a set of blocks called nodes. A node has input flows and output flows. It contains equations defining output flows in terms of input flows, local variables, and possibly intermediate states variables. These equations can be encapsulated in states of automata. They can also instantiate other nodes. Each node can be provided with a contract that defines a set of properties to be enforced on the program. These properties are enforced, at compilation time, through discrete controller synthesis [18].

Automaton consists of states, one of them being the initial state, and transitions between them. States are associated to equations that give specific values to the output flows of the automaton node. The value of an output flow must be defined at each instant. Transitions are associated to boolean expressions related to one or several input flows of the automaton node.

Figure 3 presents an automaton modelling a lamp. This automaton is contained in a node that has two input flows $(\mathrm{c} 1, \mathrm{c} 2)$ and two output flows (cmd, lum). The automaton has two states (Off, On) and two transitions. Each state is associated to two equations that give values to the output flows. The equation $\mathrm{cmd}=\mathrm{s}$ _off $\rightarrow$ nothing means that at the state off, cmd is equal to s_off (switch off) if this state is newly activated and nothing otherwise. The reason is twofold. First, the value of an output flow must be defined at each instant. Second, this prevents from continuously computing $\mathrm{cmd}=\mathrm{s} \_$of $\mathrm{f}$ while the lamp is already off. The equation $\operatorname{lum}=0$ means that at the state of $f$ the lamp provides a luminosity equal to 0 lux. The input flows $\mathrm{c} 1$ and $\mathrm{c} 2$ are boolean variables.

The initial state of the lamp automaton is Off. In this state, when $\mathrm{cl}$ is false (i.e., not $\mathrm{c} 1$ is true), the automaton goes to the state on and the output flows take the values given by the equations of this state. Otherwise (i.e., $\mathrm{c} 1$ is true), the automaton remains in the state $\mathrm{Off}$. This means that in the state off, there is an implicit transition associated to $\mathrm{C} 1$ that allows to remain in this state. In the same way, when the automaton is in the state On, if $c 2$ is true, the automaton goes to Off. Otherwise (i.e., $c 2$ is $f a l s e)$ it remains in the state On. There is an implicit transition associated to not $\mathrm{c} 2$ allowing to stay in the state On.

This node example could be designed using only one input flow to reduce the number of variables used. For instance, not $\mathrm{c} 1$ and $\mathrm{c} 2$, in the automaton transitions, could be respectively replaced with not $\mathrm{C}$ and $\mathrm{c}$.

Discrete controller synthesis (DCS) [18] is a formal method used to enforce a set of properties, called objectives, on a model. DCS is enabled by H/BZR at compilation time. Given a model that represents all the possible behaviours of a system and a set of target objectives, DCS

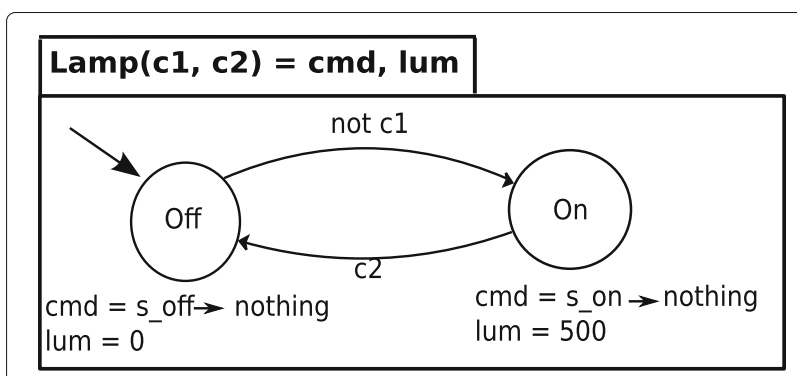

Fig. 3 Example of H/BZR node 
inhibits all the behaviours that violate the objectives. To do this, DCS requires to partition the variables of the considered model in two sets: controllable and uncontrollable variables. Once the variables are partitioned, the DCS algorithm explores the state space of the model and computes the possible values of the controllable variables. The aim is to enforce the target objectives, whatever the values of the uncontrollable variables. For instance, $\mathrm{c} 1$ and $\mathrm{c} 2$ in Fig. 3 can be defined as controllable variables to enforce an objective related to the luminosity of a room.

After the controller synthesis, several solutions can be possible regarding the objectives to achieve. For instance, the lamp can be Off or On to provide a luminosity greater or equal to 0 lux. However, one solution must be chosen. For this, the backend of the H/BZR compiler selects one of the solutions. It is possible to guide the selection with two options. Firstly, the compiler backend favours the value $t r u e$ to the value $f a l s e$ for a boolean variable. For instance, in the lamp automaton (Fig. 3), to favour staying Off, the transition from Off to on is associated to not $\mathrm{cl}$. Here, the implicit transition that remains in the state off (associated to $\mathrm{C} 1$ ) is favoured by the compiler backend.

The second option is that the compiler backend follows the declaration order of the variables and gives to them the value $t r u e$. If this does not enforce the target objectives it changes the values to false following the inverse of the declaration order. Hence, when declaring $\mathrm{c} 1$ before $\mathrm{c} 2$, if two transitions $\mathrm{T} 1$ and $\mathrm{T} 2$ respectively associated to not $\mathrm{C} 1$ and not $\mathrm{C} 2$ are possible, the compiler backend will choose T2. It gives true to $\mathrm{C} 1$ and false to $\mathrm{C} 2$ (not $\mathrm{C} 2$ is true and $\mathrm{T} 2$ is chosen).

\subsubsection{Execution of a H/BZR program}

The compilation of a H/BZR program generates a code in $\mathrm{C}$ or Java. In both cases, the generated code includes a function called step. The step takes as parameter a set of input values, computes the output values that allow to reach the target objectives, and updates the state of the automaton that models the system. One execution of the step function corresponds to one reaction of the system. Therefore, the step must be executed each time a reaction is required. Executing the step requires to ensure that the state of the automaton is always consistent with the state of the actual system. This is done by combining LINC and H/BZR.

\subsection{Combination of LINC and H/BZR}

LINC is designed to implement rules that react to events (e.g., production of a new sensor value). Hence, a LINC rule is used to execute the step function when necessary (i.e., each time an event occurs). This rule first collects data (e.g., through sensors) and then, it invokes the step in order to compute appropriate commands to send to the system (e.g., through actuators). Thanks to its transactional guarantees, LINC ensures that a group of actions are all done or none of them is done. The step is thus executed in a transaction, together with the sending of the commands. Hence, if a command cannot be sent, the step is not executed and the state of the automaton stays consistent with the state of the actual system. More details on the combination of LINC and H/BZR can be found in [29].

\subsection{Autonomic computing}

Autonomic computing [19] has been used in several solutions for the management of smart environment devices [30-33]. It consists in creating systems that manage themselves by performing self-configuration, self-optimisation, self-healing or self-protection. This is done for instance through a MAPE-K loop. In an autonomic system (cf. Fig. 4), an autonomic manager, based on knowledge, continuously

1. Monitors a managed element by collecting data;

2. Analyses the data to decide if changes are needed;

3. Plans changes based on the analysed data;

4. Executes the changes through actuators.

The knowledge consists of information related to the managed element and to its environment. The knowledge is updated when executing the changes.

\section{Framework description}

As depicted in Fig. 1, the framework consists of:

- An abstraction layer: It is based on the associative memory of the middleware LINC. The operations $r d$ and put are used to respectively read the latest value measured by any sensor (i.e., rd (sensor_id, val)) and to send a command to any actuator (i.e., put (actuator_id, command)).

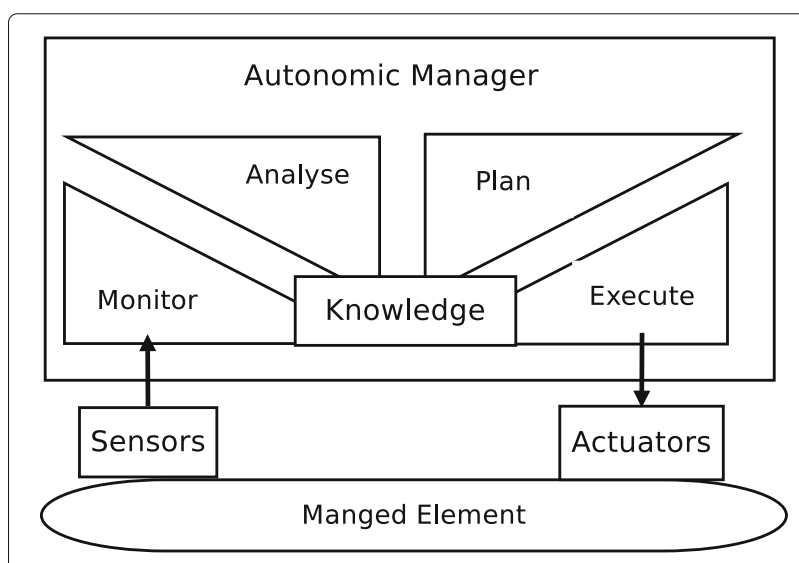

Fig. 4 Autonomic system architecture 
- An environment aware controller: It is designed through H/BZR and DCS. The controller computes appropriate commands, to reach the objectives without conflict, and is based on a generic model of the environment. This model describes the behaviour of the devices and captures environment dependencies. The model is generic in the sense that it does not describe the behaviour of specific devices (e.g., lamp_12) but types of devices (e.g., lamp). This allows to use the same controller for other environments that have the same types of devices. For instance, let us consider a controller designed for a room equipped with one lamp and one shutter. This controller can be instantiated in other rooms equipped with a lamp and a shutter with any communication technology. This controller can also be instantiated in an open-space equipped with several lamps and several shutters, all the lamps (resp. shutters) are seen as one lamp (resp. one shutter) by the controller. Finally, the controller can be reconfigured, under some conditions, to deal with changing objectives (e.g., weekdays vs. weekends).

- A transactional execution mechanism: It is based on the distributed transactions of the middleware LINC. The update of the controller state and the update of the actual system is included in the same transaction. Hence if an action cannot be performed on the actual system (e.g., due to a communication error), the controller state is not updated. Hence, the inconsistencies between the controller and the actual system are avoided.

\subsection{Autonomic management of devices}

The proposed framework enables the autonomic management of smart environment devices, through a variant of the MAPE-K loop (MIADIE-K loop). As shown in Fig. 1, the devices are monitored and the collected data are interpreted (MI). Then, an analysis is done and appropriate commands are computed $(A D)$, based on knowledge $(K)$. Finally, the commands are interpreted and sent and the knowledge is updated (IE).

- Monitoring and Interpretation (MI): Provide the data required to make decisions. Sensor data are first collected through the abstraction layer. Then, the data may be interpreted. The aim is to aggregate them, to transform them or to use them to estimate other data. For instance if a temperature data is needed and there are two temperature sensors, their average may be used as the temperature. Another example is to use the value measured by a $\mathrm{CO}_{2}$ sensor to estimate the number of people. Data transformation, aggregation and estimation are not subject to conflicts (they do not involve actuators) and thus, are performed by writing LINC rules, by the developers.
- Analysis and decision (AD): Analyse the data obtained from the monitoring and compute the commands to send to the actuators. To avoid conflicts, the commands are computed by a controller (step function) obtained through H/BZR and DCS. Nevertheless, it is possible for developers to manually write rules to achieve simple objectives (involving devices that do not affect an environment parameter for instance $\mathrm{CO}_{2}$ ). Such objectives are easy to achieve while avoiding conflicts and thus, do not require to use the controller. These rules also analyse specific monitoring data and compute commands, based on knowledge.

- Knowledge (K): The knowledge used by the controller is one instance of the generic environment model. It consists of a set of automata and equations. Each automaton describes the behaviour of a specific device by specifying its states, its transitions and its effects on the environment. The knowledge used by the LINC rules (achieving simple objectives) is a set of resources stored in bags and modelling the states of specific devices.

- Interpretation and execution (IE): Interpret the computed commands, send them and update the knowledge. The interpretation allows to send a specific command to several actuators.

\section{Framework usage by developers}

The proposed framework allows developers to

- Generate an executable model from a model of the environment and a set of target objectives;

- Create soft sensors and soft actuators respectively from physical sensors and actuators;

- Deal with changing objectives through the automatic reconfiguration of the controller;

- Consider a high number of devices through the modular design of the MIADIE-K loop;

- Write LINC rules to manually achieve simple objectives, involving a small number of devices, that do not lead to conflicts.

\subsection{Generating an executable model}

Developers design a H/BZR program by defining a model of the considered environment, the target objectives and the controllable variables. Then, the framework generates an executable model that manages the devices of the environment and achieves the objectives.

\subsubsection{Modelling the environment}

Developers first consider a set of parameters of the environment and identify all the devices that affect at least one parameter. Then, developers model each type of identified device as an automaton contained in a H/BZR node. 
The automaton modelling a device type specifies the different states of the device type, its state transitions, the environment parameters it affects and how it affects them. Modelling types of devices, instead of specific devices, allows for the models re-usability.

Let us consider as an example, in the context of building automation, a room equipped with a shutter and a lamp. To model this room, two parameters (luminosity and air) are first considered. Then, two automata are designed to model a shutter and a lamp.

Figure $5 \mathrm{a}$ presents the automaton that models a shutter. This automaton is contained in a H/BZR node that has two input flows (c, o_lum) and three output flows (cmd, lum, air). The automaton has two states (Closed, Opened) and two transitions. Each state is associated to three equations to produce the command of the shutter (cmd) and specify its effects on the environment (lum, air). In the state Closed, the command is equal to close (resp. nothing) if this state is (resp. not) newly activated. This prevents from continuously computing the command close while the shutter is already Closed. In this state, the shutter provides a luminosity equal to zero (lum $=0)$ and does not allow outdoor air to pass $(\operatorname{air}=$ false). In the state Opened, the shutter provides a luminosity equal to the outdoor luminosity ( 1 um = o_lum) and allows outdoor air to pass (air = true). The transitions going from a given state to a different one are associated to not $c$. This allows to open or close the shutter only when necessary. Figure $5 \mathrm{~b}$ presents the lamp automaton. This automaton is the same than the one presented in Fig. 3.

\subsubsection{Defining the target objectives and the controllable variables}

Developers design a main H/BZR node that instantiates the automata modelling the devices and has a contract part. Then, developers define in the contract, the target objectives and the controllable variables.

Defining the objectives Developers specify the values that the considered environment parameters must take. These values may depend on data collected from the environment. This is done using variables and operators. Variables are used to refer to the collected data and also to the environment parameters. Operators, for instance, $\Rightarrow$ (logical implication) and $\wedge$ (and) are used to express the relations between the variables. Examples of objectives for a room are:

1. presence $\Rightarrow$ luminosity in $[500,600]$ lux;

2. presence $\Rightarrow$ noise $<80 \mathrm{~dB}$;

3. presence $\wedge$ temperature $<17^{\circ} \mathrm{C} \Rightarrow$ heat;

4. presence $\wedge \mathrm{CO}_{2}>800 \mathrm{ppm} \Rightarrow$ ventilation.

The first objective means that if a presence is detected, in the room, the luminosity must be between 500 and 600 lux. The second objective means that if a presence is detected, the noise level must be lower than $80 \mathrm{~dB}$. The third objective (resp. the fourth objective) means that if a presence is detected and the temperature is below $17^{\circ} \mathrm{C}$ (resp. the $\mathrm{CO}_{2}$ is above $800 \mathrm{ppm}$ ), the room must be heated (resp. ventilated). The devices required to reach the objectives and the actions to perform on them will be decided by the step function. For instance, the first

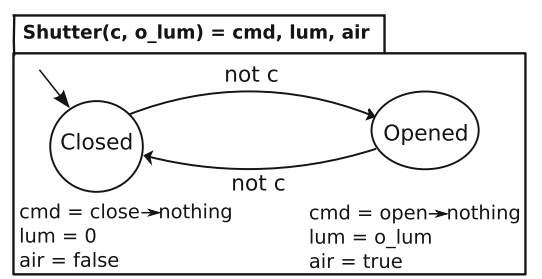

(a)

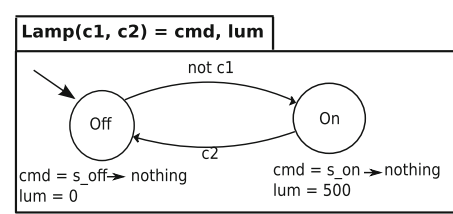

(b)

\begin{tabular}{|l|}
\hline Room(i_presence, o_lum $)=$ shutter_cmd, lamp_cmd \\
\hline contract enforce \\
i_presence $\Longrightarrow$ lum in $[500,600]$ \\
with (c1_lamp, c2_lamp,c_shutter) \\
(lamp_cmd, lum_lamp) = Lamp $(\mathrm{c} 1$ lamp, c2_lamp); \\
(shutter_cmd, lum_shutter, air $)=$ Shutter(c_shutter, o_lum); \\
lum =lum_lamp + lum_shutter;
\end{tabular}

(c)

Fig. $5 \mathrm{H} / \mathrm{BZZR}$ program of the room example. a Shutter node, $\mathbf{b}$ Lamp node, $\mathbf{c} H / B Z R$ contract 
objective can be reached by switching on the lamp or opening the shutter. The second action may be preferred for energy savings.

Defining the controllable variables Developers first analyse the input flows of the nodes that contain the automata modelling the devices. The aim is to identify the input flows that are controllable (their values are not given by the monitoring). Then, developers declare the identified input flows, in the contract part of the main H/BZR node, as controllable variables. For instance, in the lamp automaton, presented in Fig. 5b, the input flows $\mathrm{c} 1$ and $\mathrm{c} 2$ are controllable variables. In the shutter automaton, presented in Fig. 5a, the input flow $\mathrm{C}$ is a controllable variable. The input flow o_lum represents the value measured by an outdoor luminosity sensor and hence it is uncontrollable.

Developers can use the declaration order of the controllable variables to express preference between the transitions of the different automata. A transition $\mathrm{T} 1$ associated not $\mathrm{C} 1$ is preferred to another transition $\mathrm{T} 2$ associated to not $\mathrm{c} 2$ if is $\mathrm{c} 1$ is declared after $\mathrm{c} 2$.

\subsubsection{Example of $H / B Z R$ program for a room}

Let us consider again the room, equipped with a shutter and a lamp. The objective to achieve is to maintain the luminosity between 500 and 600 lux when a presence is detected while minimising the energy consumption (i.e., prefer natural lighting to artificial lighting).

The H/BZR program defined for this room is presented in Fig. 5. It consists of three nodes. The first two nodes respectively contain the shutter and the lamp automata (Fig. 5a and b). The third node, presented in Fig. 5c, is the main node. It has two input flows (i_presence and o_lum) and two output flows (shutter_cmd and lamp_cmd). The input flows respectively represent the values measured by the room indoor presence sensor and outdoor luminosity sensor. The output flows respectively correspond to the commands to send to the shutter and to the lamp. This node defines a contract to enforce the target objective, through DCS, with the controllable variables c1_lamp, c2_lamp and c_shutter. These controllable variables are respectively related to the lamp and the shutter. The luminosity objective is expressed as follows: i_presence $\Rightarrow$ lum in $[500,600]$ where lum is equal to the sum of the luminosity provided by the shutter and the lamp. The controllable of the shutter is declared after those of the lamp to specify that natural lighting is preferred to artificial lighting.

\subsubsection{Executable model generation}

Figure 6 shows how from the generic environment model, the target objectives and the controllable variables (i.e., a H/BZR program designed by developers) are generated the following elements:
1. A step function;

2. A LINC rule template;

3. An instance of the LINC rule template.

At runtime, the LINC rule instance invokes the step function each time a relevant event occurs. When invoked, the step computes and returns appropriate commands that will be executed by the rule instance.

Step generation The step is generated through the compilation of the H/BZR program designed by developers. For instance, the compilation of the H/BZR program presented in Fig. 5 generates a step.

LINC execution rule template generation The execution rule template is generated from the H/BZR program and the objects of the PUTUTU framework. This rule template collects the inputs of the main H/BZR node, invokes the step function with the collected inputs and sends the computed commands (outputs of the main H/BZR node). The precondition of the rule template consists of a set of $r d$ operations: one $r d$ for each input of the main H/BZR node and one $r d$ to invoke the step. The performance of the rule template consists of a sequence of two transactions. The first transaction consists of a set of $r d$ operations to verify if the collected inputs have not changed and a set of put operations to send the computed commands and update the knowledge. One put is generated for each output of the main H/BZR node and one put is generated to update the knowledge. The second transaction consists of a set of $r d$ operations to verify if the collected inputs did not change, one $r d$ to verify if the knowledge did not change and one put operation to signal an actuator problem to the maintenance team. In the second transaction, the fact that the collected inputs are still the same and the knowledge did not change means that the first transaction aborted when performing an action on an actuator (it is faulty or unreachable). Otherwise, the knowledge would be updated. Hence, the maintenance is informed if the inputs are the same and the knowledge did not change.

Listing 2 presents the rule template generated from the H/BZR program presented in Fig. 5. The precondition of this rule first reads the value measured by an indoor presence sensor in the variable i_pres_id_val. This is done by applying a $r d$ on the Sensors bag of a PUTUTU object. Then, at line 2 , the precondition reads the value measured by an outdoor luminosity sensor in the variable o_lum_id_val. At line 3, the precondition stores the read sensor values in one variable called inputs using the LINC operation IC (i.e., INLINE_COMPUTE). In LINC, the $I C$ operation executes any python code and returns a tuple of string (here containing only one 


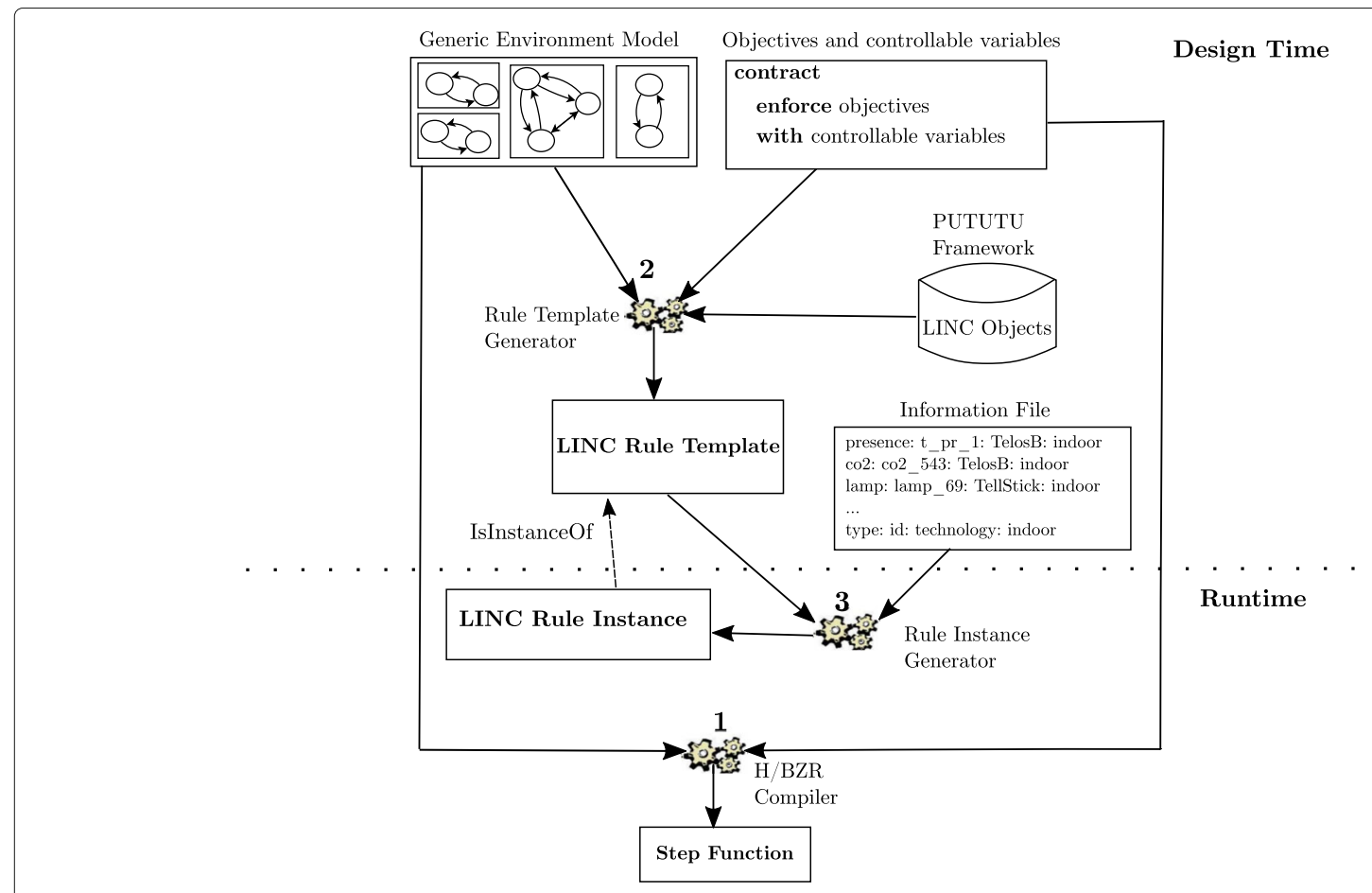

Fig. 6 Executable model generation

value). Finally, the precondition invokes the step function (line 4) and stores the computed commands in the variables shutter_cmd and lamp_cmd (line 5). These variables will be used in the first transaction of the performance.

The first transaction verifies if the sensors data did not change (lines 8,9 ). Then, it sends the computed shutter command to a shutter actuator. This is done by applying a put on the Actuators bag of a PUTUTU object. This transaction also sends the computed lamp command to a lamp actuator (line 11) and updates the knowledge (line 12). If a command cannot be sent due to a problem on the shutter (resp. the lamp) actuator, the put at line 10 (resp. line 11) fails and the knowledge is not updated. This prevents from inconsistencies. To signal the shutter or the lamp problem, the second transaction, sends a SMS to the maintenance team.

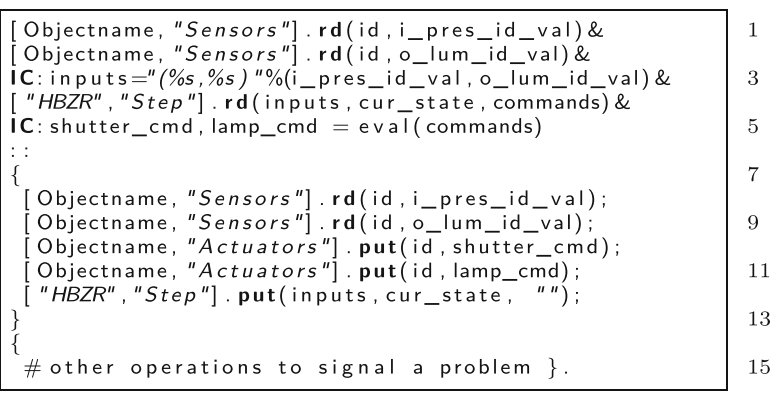

Listing 2 Generated execution rule template example
LINC execution rule instance generation From the generated rule template, a rule instance is generated with the actual sensors and actuators. This is done using a file that contains information related to the considered environment devices (i.e., type, id, technology, location). To generate an instance of the execution rule template, the rule instance generator replaces:

- In rd operation, Objectname and id by the technology and the id of the corresponding sensor;

- In put operation, Objectname and id by the technology and the id of the corresponding actuator.

Listing 3 presents the file that describes the devices of the room example. This file specifies that the room is equipped with a TelosB indoor presence sensor with an id equal to pr1, a RFXCOM outdoor luminosity sensor, an EnOcean lamp actuator and a KNX shutter actuator.

\begin{tabular}{|l|l|}
\hline \# type: id: technology: location & 1 \\
presence: pr1: TelosB: indoor & \\
luminosity: Iu $9:$ RFXCOM: outdoor \\
lamp:e_l_1: EnOcean: indoor \\
shutter: shutter_43: KNX: indoor
\end{tabular}

Listing 3 Information file example

Listing 4 presents the rule instance generated from the execution rule template of Listing 2 and the description file presented in Listing 3 . This rule reads the values of 
specific sensors, invokes the step and sends the computed commands to specific actuators.

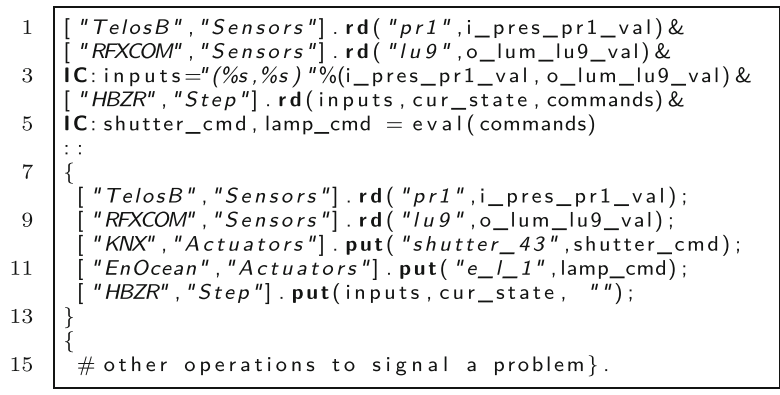

Listing 4 Generated execution rule instance example

\subsection{Creating soft sensors}

Developers can create soft sensors from physical sensors to aggregate the data collected from the environment, to transform them or to estimate other data. For that, they write specific LINC rules called monitoring rules. These rules do not involve actuators and cannot be conflicting. These rules rely on the abstraction layer which hides the devices heterogeneity. Developers can also write monitoring rules for other data sources (e.g., agenda) to transform data or estimate required data.

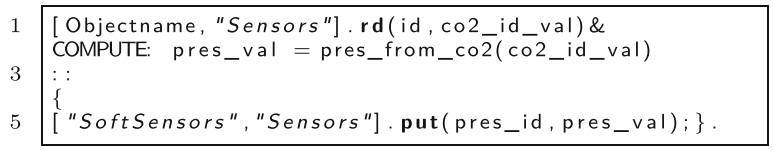

Listing 5 Monitoring rule template example

Listing 5 presents an example of monitoring rule template. This rule template creates a soft presence sensor from a physical $\mathrm{CO}_{2}$ sensor. The precondition of this rule first reads in the variable $\mathrm{CO}_{2}$ id_val, the value measured by a $\mathrm{CO}_{2}$ sensor. Then, the precondition invokes a function (i.e., pres_from_co2) with the variable $\mathrm{CO}_{2}$ id_val as parameter to estimate if a presence is detected or not. The result is stored in the variable pres_val. The performance inserts the resource (pres_id, pres_val) in the Sensors bag of the SoftSensors LINC object. This specifies that a presence has been detected or not by the soft presence sensor with the id pres_id. This monitoring rule is triggered each time a new $\mathrm{CO}_{2}$ value is produced.

Once designed, a monitoring rule template is instantiated for a specific environment and the created soft sensor is added in this environment information file. Soft sensors and physical sensors are handled similarly when instantiating the step execution rule template.

\subsection{Creating soft actuators}

Developers can create soft actuators to allow some computed commands to be sent to more than one actuator. A soft actuator groups several actuators, that have different ids and possibly different communication technologies, as one actuator. When creating a soft actuator, developers specify the number of actuators that must perform the action corresponding to the command (e.g., three lamps must be switched on).

When used in a rule, a soft actuator is seen as any other actuator: a put is done on its Actuators bag. When applied, the put sends the specified command to all the physical actuators that are associated to the soft actuator. The put succeeds if the action corresponding to the command can be performed by the specified number of actuators. Otherwise, the put fails.

Creating a soft actuator consists first in creating a specific PUTUTU object that encapsulates one or several technologies. This is straightforward and is done by inheriting from the existing PUTUTU objects that encapsulate the technologies of the target physical actuators. By default, the operation put(id,command) on a PUTUTU object Actuators bag sends the command, given as parameter, to only one actuator. Therefore, it is required to modify the behaviour of the put operation, on the created object Actuators bag, to send the command to the required number of actuators.

Once defined, a soft actuator is added in the environment information file and will be used by the rule that invokes the step, as if it were a physical actuator.

\subsection{Dealing with changing objectives}

Controlling smart environment devices requires to deal with changing objectives. The reason is that a realistic environment can have different configurations with different objectives (e.g., working time and holidays in a building). This is done by first designing a controller for each configuration. Then, switching between the controllers, at runtime, as illustrated in Fig. 7.

Let us consider a system and a set of controllers, designed in H/BZR, to achieve different objectives. To achieve its objectives, each controller accepts specific states of the system and rejects the other states (i.e., those that can violate its objectives). A state rejected by a given controller is not allowed to be reached when this controller is activated. Hence, switching an activated controller (e.g., $C t_{1}$ ) for another controller (e.g., $\left.C t_{2}\right)$ is possible only if the current state of the system (accepted by $C t_{1}$ ) is accepted by the controller $\mathrm{Ct}_{2}$.

Switching controllers is not an easy task in general because it is not straightforward to decide if a given state is accepted by a controller. Indeed, a state is accepted by a controller if it:

- Belongs to the state space (set of known states) of the controller;

- Does not violate an objective of the controller; 


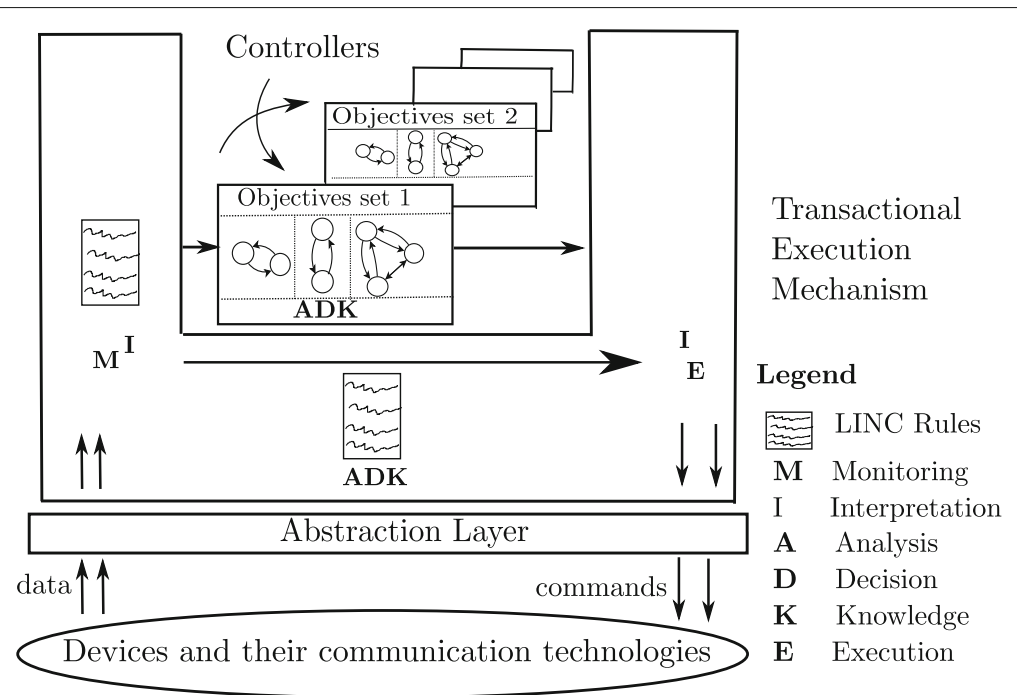

Fig. 7 Controller reconfiguration through the framework

- Does not lead, through one or several uncontrollable transitions, to a state violating an objective.

However, in the context of smart environments, particular solutions can be performed to enable the switch of controllers. An example of solution is to design the different controllers in such a way that they all have the same initial state. This allows to switch them when this state is reached. For instance, a building may have a night state where it is not occupied, completely closed, not heated and not ventilated. This state can be use as the initial state of all the building controllers. Another possible solution is to try to synthesise the target controller from the current state of the activated controller. If the controller synthesis succeeds and the current state has not changed, the switch can be done.

To enable the reconfiguration, the execution rule of each controller is associated to a resource to be activated or deactivated. For this, a LINC bag called CurConfiguration is first created. This bag contains one resource indicating the current configuration of the considered environment. Then, each execution rule is modified to enable its activation and deactivation.

To illustrate the reconfiguration, let us consider an example in the context of building automation. One can design two controllers (with the same initial state), for different configurations of a building (e.g., working time and holidays). Then, the execution rules of the controllers are modified as shown in Listing 6 for the working time execution rule. A $r d$ on the configuration of the controller is added in the beginning of the precondition and in the beginning of each transaction. This ensures that an execution rule is triggered only if the resource corresponding to its controller configuration is present in the bag CurConfiguration.
Hence, switching a controller for another one consists in removing the resource of CurConfiguration and adding the appropriate resource. This is done by writing a switching rule. For instance, Listing 7 presents an example of LINC rule that switches the working time controller for the holidays one when the event holidays_start is triggered and the night state of the building is reached. The performance of the rule consumes the event resource and the current configuration resource and inserts the Holidays resource.

When the controllers are numerous, instead of writing switching rules that may be conflicting (activate and deactivate a controller at the same instant), one can design, using H/BZR, a switching controller. This controller will decide the actions to perform on the controllers (activate, deactivate) and avoid conflicts.

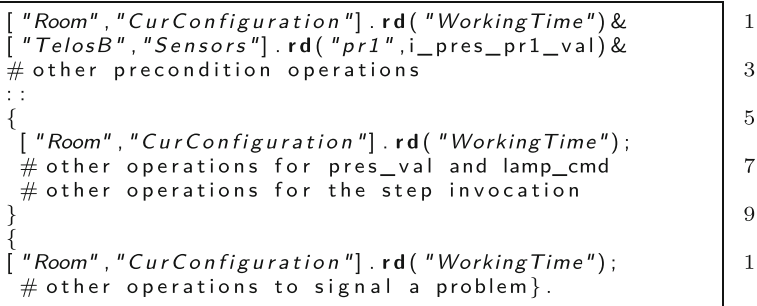

Listing 6 Modified execution rule example

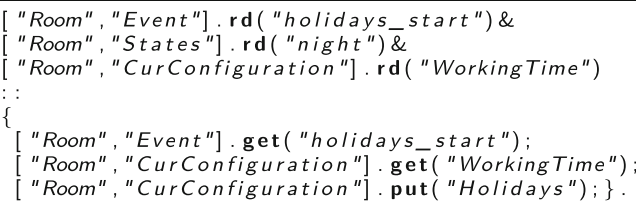

Listing 7 Switching rule example 


\subsection{Deployment}

After the design phase, the framework generates a set of LINC rules and objects that have to be deployed. The objects consist of one HBZR object (used for the step and several PUTUTU objects, one for each sensors/actuators technology used in the target environment. Each PUTUTU object (e.g., Plugwise) must be deployed on a computing device that has the appropriate dongle (e.g., plugwise dongle). The HBZR object does not require a dongle and can be deployed on any computing device. Once the deployment is performed, the control loop starts and the controller will be invoked when a relevant event occurs in the environment.

\subsection{Handling a high number of devices}

When the number of devices is high, using one single loop may become a bottleneck. First, the synthesis of the loop controller can take a lot of time or not succeed due to computing resource limitations. Second, the execution rule reads several data in its precondition part. This can lead to runtime performance degradation.

In this case, the devices can be partitioned in several sets. Then, one loop is used for each set, as illustrated in Fig. 8. In this context, each set of devices has a controller. If the devices sets are independent, nothing more is required. Otherwise their controllers must be coordinated, using priorities on their actions on shared devices. In this case, an output value of a given controller can be an uncontrollable input for another one.

The coordination of controllers is currently done in LINC. The execution rule of a controller can insert a value in specific bags or read a value inserted by another controller execution rule. The coordination could also be done in H/BZR, by exploiting the potential of modular discrete controller synthesis, as done in [34].

\section{Case study}

This section illustrates the proposed framework through a case study taken in the field of building automation. The aim is to manage the devices of a building in order to achieve a set of objectives. The building is first described. Then, its devices are managed and a demonstrator is presented to show that the framework was able to reliably achieve the target objectives. Then, the management cost is evaluated to show the scalability of our approach. Finally the case study is discussed to compare the framework to related work approaches.

\subsection{Building description}

The considered building consists of ten small offices and twelve big offices, that are separated. A small office consists of a room that contains: a window, a shutter, a door, a lamp, a reversible air-conditioner (RAC), a mechanical ventilation $(\mathrm{MV})$, a temperature sensor and a $\mathrm{CO}_{2}$ sensor. A big office has an additional lamp, window, shutter and temperature sensor compared to a small office. A big office also has a presence sensor. Several sensors are installed outside the building to enquire outdoor conditions (i.e., luminosity, $\mathrm{CO}_{2}$, noise, temperature, pollen). Noise sensors are also installed in the corridors. The actuators and sensors of the building use different communication technologies (e.g., EnOcean, TelosB). Each room has a file that describes its devices (id, type, technology and location). Information about the meetings (e.g., day, time, features) that will be held in each room can be obtained through a specific agenda. The devices of the rooms must be managed to achieve the following objectives:

- For comfort, when a presence is detected, the luminosity must be between 500 and 600 lux and the noise level must be lower than $80 \mathrm{~dB}$;

- For air quality, when a presence is detected and the $\mathrm{CO}_{2}$ exceeds $800 \mathrm{ppm}$, the room must be ventilated. It must not be be polluted by pollen or outdoor $\mathrm{CO}_{2}$ and must be quickly ventilated between meetings separated by less than 30 minutes;

- For comfort, when a presence is detected and the temperature is below $17^{\circ} \mathrm{C}$ (resp. above $27^{\circ} \mathrm{C}$ ), the room must be heated (resp. cooled);

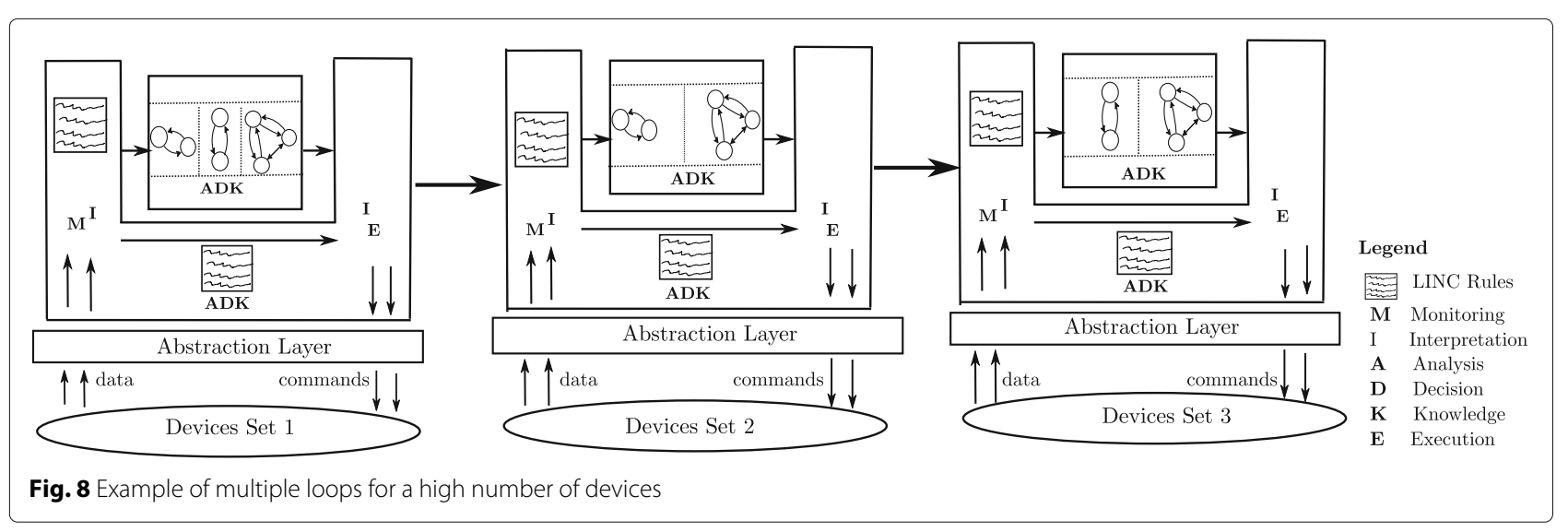


- For confidentiality, the room must be completely closed during a confidential meeting;

- For energy savings, natural lighting, ventilation, heating and cooling are preferred to artificial lighting, ventilation, heating and cooling.

\subsection{Devices management using the proposed framework}

To achieve the objectives in the rooms, developers have to design a H/BZR program, from which a step function and an execution rule template will be generated. Then, developers have to write two monitoring rule templates to respectively estimate a presence in a small room (not equipped with a presence sensor) and to compute a temperature average in a big room (has two temperature sensors). Developers also have to write a monitoring rule template to know from an agenda, if there is a meeting or not and if a meeting will be held in less than $30 \mathrm{~min}$, after a previous meeting. Finally, the rule templates are instantiated in each room.

\subsubsection{Designing a H/BZR program}

This requires to design a generic model of a room. For this, a set of environment parameters are first considered (i.e., luminosity, noise, $\mathrm{CO}_{2}$, temperature, pollen and air). Then, the effects of the devices on these parameters are specified. As presented in Table 1:

- A window: affects five parameters (noise, $\mathrm{CO}_{2}$, air, temperature, pollen). When the window is opened, it introduces the outdoor noise in the room. It can ventilate, heat or cool the room, depending on the outdoor conditions. It can pollute the room by introducing pollen or outdoor $\mathrm{CO}_{2}$;

- A shutter: affects two parameters (luminosity, air). When the shutter is closed, it provides a luminosity equal to zero and stops the outdoor air;

- A door: affects five parameters (luminosity, noise, $\mathrm{CO}_{2}$, temperature, air). When the door is opened, it lights the room (if the corridor lamps are on) and introduces the corridor noise;

- A lamp: affects one parameter (luminosity). It provides 500 lux when it is on and 0 when off;

- A RAC: affects the temperature of a room;

- A MV: affects the $\mathrm{CO}_{2}$ and the air.

Table 1 Environment parameters and devices

\begin{tabular}{lllllll}
\hline & Window & Shutter & Door & Lamp & RAC & MV \\
\hline Luminosity & & $\times$ & $\times$ & $\times$ & & \\
Noise & $\times$ & & $\times$ & & & \\
$\mathrm{CO}_{2}$ & $\times$ & & $\times$ & & & $\times$ \\
Temperature & $\times$ & & $\times$ & & $\times$ & \\
Pollen & $\times$ & & & & & \\
Air & $\times$ & $\times$ & $\times$ & & & $\times$ \\
\hline
\end{tabular}

A generic room model is obtained by designing automata that describe the behaviours of a window, a shutter, a door, a lamp, a RAC and a MV. All the automata have an output flow that is the command to send to the modelled actuator. This command is equal to nothing when the actuator should not receive a command. For instance, to switch off a lamp, the command is equal to s_off when the lamp is on and nothing as long as it remains off. The lamp and shutter automata are those presented in Fig. 5.

Figure 9 presents the automaton that describes a door. This automaton has two states and two transitions. Each state is associated to two equations to produce the command of the door and also specify its effect on a room noise level. For instance, at the state Closed, the door affects the noise level with a value equal to zero. The effects of the door on the other parameters (i.e., air, $\mathrm{CO}_{2}$, temperature, luminosity) are not considered because the corresponding sensors do not exist in the corridor and their values cannot be obtained. The transitions that go from a state to a different one are associated to not $\mathrm{C}$. This allows to open or close the door only when necessary.

Figure 10 presents the automaton that describes the behaviour of a reversible air-conditioner (RAC). This automaton has three states and six transitions. Each state is associated to three equations to produce the command of the RAC and also specify its effects on the room. For instance, at the state Off, the RAC does not cool nor heat the room. This automaton is contained in a node that has two input flows $\mathrm{C} 1$ and $\mathrm{c} 2$. The reason is that, at each state, three transitions can be triggered (i.e., two transitions that leaves the state and one that allows to stay). To associate a different boolean expression to each of the three transitions of a state, at least two variables are needed. For instance, when the state off is activated, if the input flow $\mathrm{Cl}$ is false, the RAC automaton goes to the state Cooling. If $\mathrm{c} 2$ is false, it goes to the state Heating. If both $\mathrm{c} 1$ and $\mathrm{c} 2$ are true, it remains at the state off. Finally, if both $\mathrm{c} 1$ and $\mathrm{c} 2$ are false, at the same instant, the transition that was first declared is

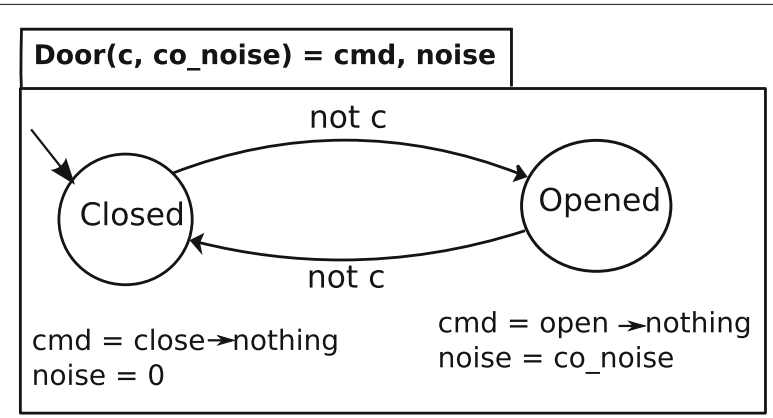

Fig. 9 Door automaton 


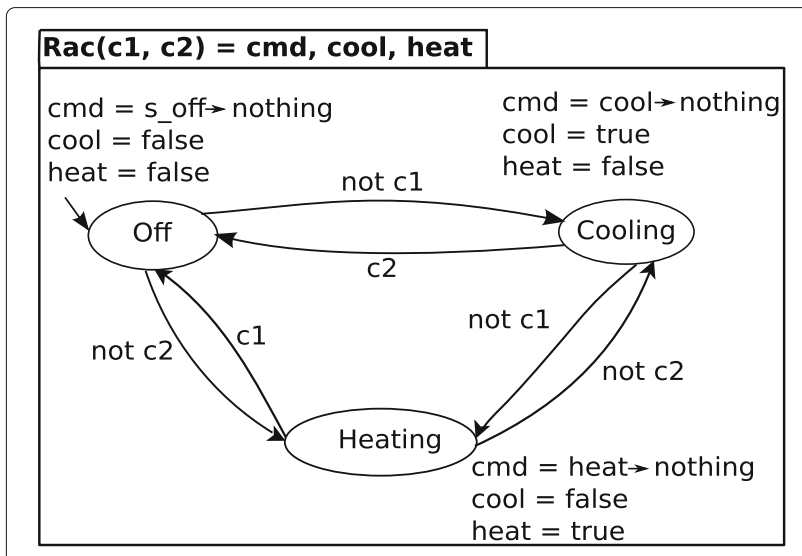

Fig. 10 RAC automaton

chosen. Associating not $\mathrm{c} 1$ and not $\mathrm{c} 2$ (resp. $\mathrm{c} 1$ and c2) to the transitions that leave (resp. come to) the state Off means that it is preferred to maintain the RAC off for energy savings.

Figure 11 presents the automaton that describes the behaviour of a mechanical ventilation (MV). This automaton has three states. Each state is associated to three equations to produce the command of the MV and specify its effect on the $\mathrm{CO}_{2}$ concentration of a room. For instance, at the state $\mathrm{Off}$, the MV does not ventilate the room. At Mode1, the MV ventilates the room but not quickly, as done in Mode2. The transitions that leave (resp. come to) the state off are associated to not $\mathrm{cl}$ and not $\mathrm{c} 2$ (resp. $\mathrm{c} 1$ and $\mathrm{c} 2$ ) to express that it is preferable to not use the MV.

Figure 12 presents the automaton that describes the behaviour of a window. This automaton has two states. Each state is associated to five variables to specify the effects of the window on different parameters of a room. At the state Closed, the window does not ventilate, heat,

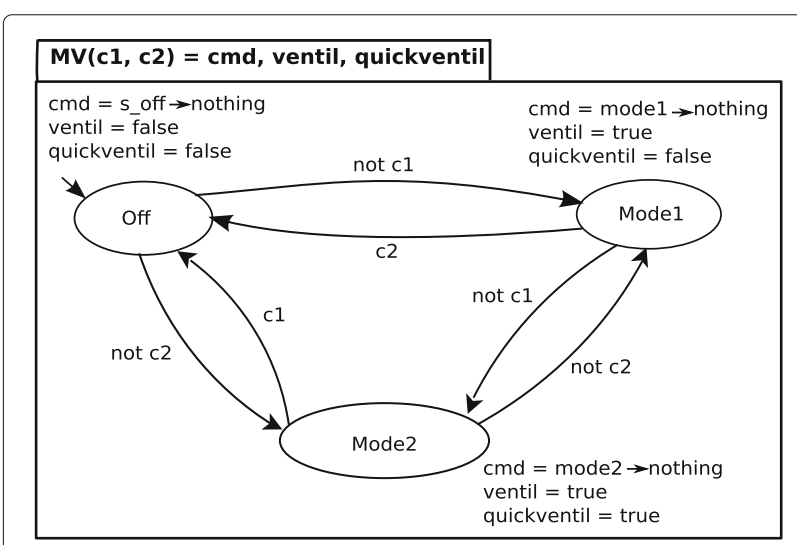

Fig. $11 \mathrm{MV}$ automaton

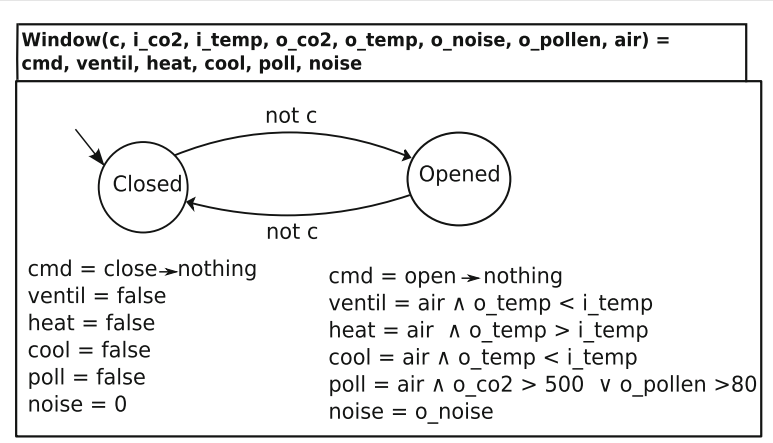

Fig. 12 Window automaton

cool, pollute nor introduce outdoor noise in a room. At the state Opened, the window can heat, cool, ventilate, pollute or affect the noise level of a room, depending on the indoor and the outdoor conditions. The transitions that lead to a different state are associated to not $\mathrm{c}$ to specify that the window should be opened or closed only when necessary.

Once the devices modelled as automata, the H/BZR node presented in Fig. 13 is designed. This node defines one instance of each device automaton and has a contract. The contract defines the target objectives and a set of controllable variables. These variables are the input flows associated to automata transitions with a value not given by the monitoring. The energy savings objective (i.e., natural lighting, heating, ventilation and cooling are preferred) is expressed by declaring the controllable variables of the shutter and the window after those of the lamp, the RAC and the MV. This H/BZR node represents a room automaton. It takes as input sensor values and meetings information and returns the commands to send to the actuators.

Once defined, the H/BZR program (set of defined nodes) was compiled to generate a step function. The H/BZR program was also used to generate an execution rule template that was instantiated for each room.

\subsubsection{Execution rule template generation}

The H/BZR program and the objects of the PUTUTU framework were used to generate an execution rule template. This rule is presented in Appendix (Listing 9). The precondition first applies nine $r d$ operations on the Sensors bags of nine PUTUTU objects, referred as Objectname, to read the sensor values (e.g., presence, $\mathrm{CO}_{2}$ ). Then, the precondition applies a $r d$ on a bag that encapsulates an agenda to obtain information related to meetings. Finally, the precondition invokes the step with the sensor values and the meetings information to compute actuators commands. The performance of the rule template consists of two transactions. The first 


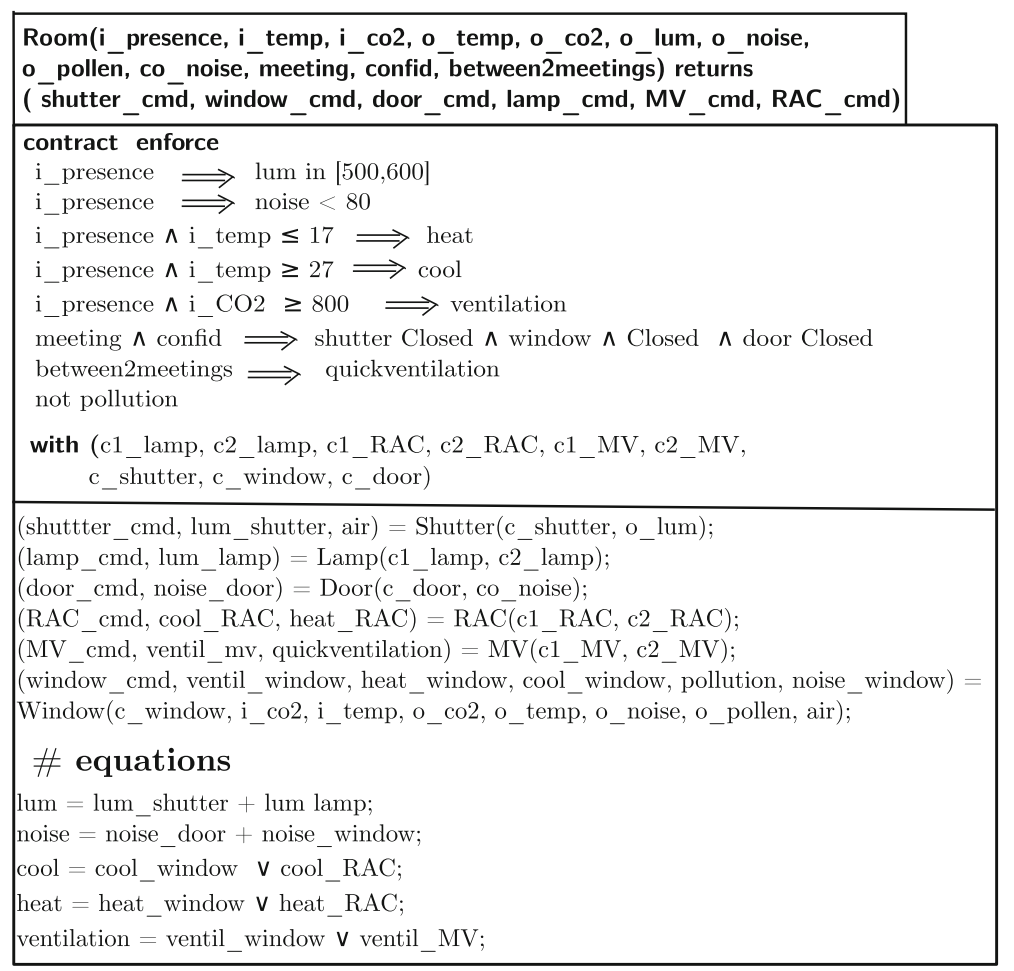

Fig. 13 Room automaton

transaction verifies if the sensor values did not change. Then, it applies six put operations on the Actuators bags of six PUTUTU objects, referred as Objectname, to send the computed command to the actuators. Finally, it updates the room generic model (changes the room automaton state). The second transaction sends a SMS to the maintenance if a command cannot be sent (e.g., communication error, actuator failure). The execution rule template was instantiated in each room of the building. For the big rooms, equipped with two lamps, two windows, two shutters, one RAC, one door and one MV, some computed commands (i.e., lamp_cmd, window_cmd, shutter_cmd) are sent to two actuators.

\subsubsection{Monitoring rules design}

A monitoring rule template is designed to estimate a presence from the value measured by a $\mathrm{CO}_{2}$ sensor, in a small room. This rule template is the one presented in Listing 5 . Another monitoring rule template is designed to compute a temperature average in a big room (is equipped with two temperature sensors). This rule is presented in Listing 8 . The precondition of this rule first reads two temperature values measured by two different sensors. Then, it uses a function to compute the average and stores it in the variable temp_aver. The performance of this rule stores the computed average in the Sensors bag of the SoftSensors object. A monitoring rule template is designed to obtain relevant meeting information from a room agenda. This rule returns a resource that specifies if there is a meeting, if it is confidential and if meeting will be started in less than $30 \mathrm{~min}$, after a previous meeting.

The designed monitoring rule templates were instantiated in specific rooms. This was done by replacing, in each operation, Objectname and id, respectively, with the technology and id of the corresponding sensor.

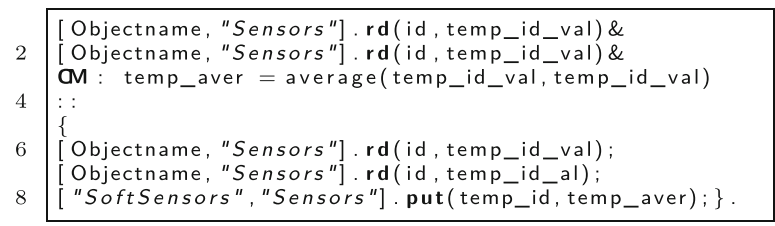

Listing 8 Temperature average rule

\subsection{Demonstrator with concrete devices}

To illustrate the framework, a demonstrator was built. The aim is to achieve, in a room, two objectives: presence $\Rightarrow$ luminosity in $[500,600]$ lux, confidential meeting $\Rightarrow$ room completely closed. The demonstrator, as shown in Fig. 14, consists of:

- A Plugwise circle [35]: Is a plug used to automatically switch on or off the lamp. This is done 


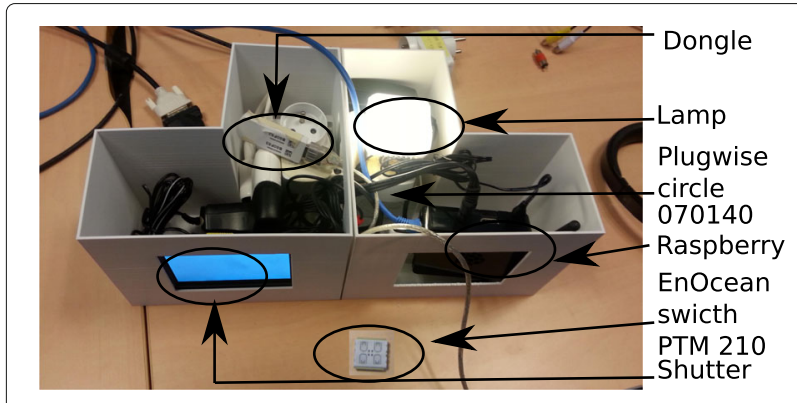

Fig. 14 Demonstrator

by applying the operation put(id,command) on the Actuators bag of a Plugwise PUTUTU object.

- An EnOcean switch [36]: Is used as a presence sensor. The switch has a button that can be pressed to emulate a presence. The value of the switch (presence detected or not) is obtained by applying the operation rd(id, value) on the Sensors bag of the EnOcean PUTUTU object.

- A graphical interface: Is used to emulate a shutter. A bag, contained in an object (Shutter), is created to send a command to the shutter. The insertion of a resource in this bag, shows the corresponding action (open, close) on the interface.

- A Raspberry Pi: Is used to deploy the objects and the execution rule. It is connected to the switch and to the circle through two dongles.

Two bags (i.e., OutdoorLuminosity and Agenda) contained in an object (Room) were created to respectively emulate an outdoor luminosity sensor and an agenda for meetings. These bags were manually filled. The step function generated for the building was used.

Figure 15 presents the MIADIE-K loop that was set up for the demonstrator. This loop is an instantiation of the the generic loop presented in Fig. 1. Data are first collected through the abstraction layer: the Room object (outdoor luminosity and confidential meeting) and the EnOcean object (presence detected by the switch). Then, the collected data are used to invoke the step function that, based on the automata of the shutter and the lamp, computes and returns the commands that achieve the objectives without conflict. Finally, the computed commands are sent to the devices and the states of the two automata are changed.

Several scenarios were performed to validate the demonstrator. Some scenarios were with a potential conflict (i.e. presence detected, outdoor luminosity in [500, 600] lux and confidential meeting held), communication errors or actuator failure (e.g., circle unplugged). In all cases, there was no conflict and no inconsistency. Three examples of scenarios are:
- First scenario: The button of the switch was pressed to emulate a presence and the outdoor luminosity was set to 500 lux by inserting the resource ("500") in the bag OutdoorLuminosity. A confidential meeting was also emulated by inserting the resource ("confidentialMeeting") in the bag Agenda. This switched on the lamp and closed the shutter. The conflict which consists in opening the shutter for daylight and closing it at the same instant for confidentiality was avoided.

- Second Scenario: It was performed just after the first scenario. The presence was still detected, the outdoor luminosity was equal to 500 lux, the shutter was closed and the lamp was on. In this context, the end of the confidential meeting was emulated by removing the resource ("confidentialMeeting") from the bag Agenda and inserting the new resource ( "notConfidentialMeeting"). This opened the shutter and switched off the lamp to save energy.

- Third Scenario: It was performed after the second scenario. The presence was still detected, the lamp was off and the shutter was opened. In this context, the outdoor luminosity was set to 700 lux. In addition, a failure was emulated on the lamp (i.e., the circle was unplugged). In this case, the controller decided to close the shutter and switch on the lamp to maintain the luminosity between 500 and 600 lux. Since there was a problem on the lamp, nothing was done and a SMS was sent to the maintenance to signal the problem. Hence, the inconsistency which consists in wrongly assuming that the lamp was switched on was avoided.

\subsection{Evaluation of the devices management cost}

This section evaluates the design cost and the runtime cost of the proposed devices management approach.

\subsubsection{Design cost evaluation}

When using the proposed framework, developers describe the actuators and define the target objectives. They do not have to manually specify the desired behaviour of the considered smart environment. This is enabled by the generation of a controller, through Discrete Controller Synthesis (DCS). For instance, in the case study, one controller was generated using six automata with a total of 65 variables, modelling a lamp, a shutter, a window, a door, a RAC and a MV. The synthesis of the controller took $1.4 \mathrm{~s}$, on a computer with a processor Intel i7 (3.4 GHZ) and $16 \mathrm{~GB}$ of RAM.

The controller synthesis algorithm is exponential in the number of variables that are used in the model that describes the devices [18]. These variables correspond to the variables that are associated to the states of the automata, modelling the devices, the controllable 


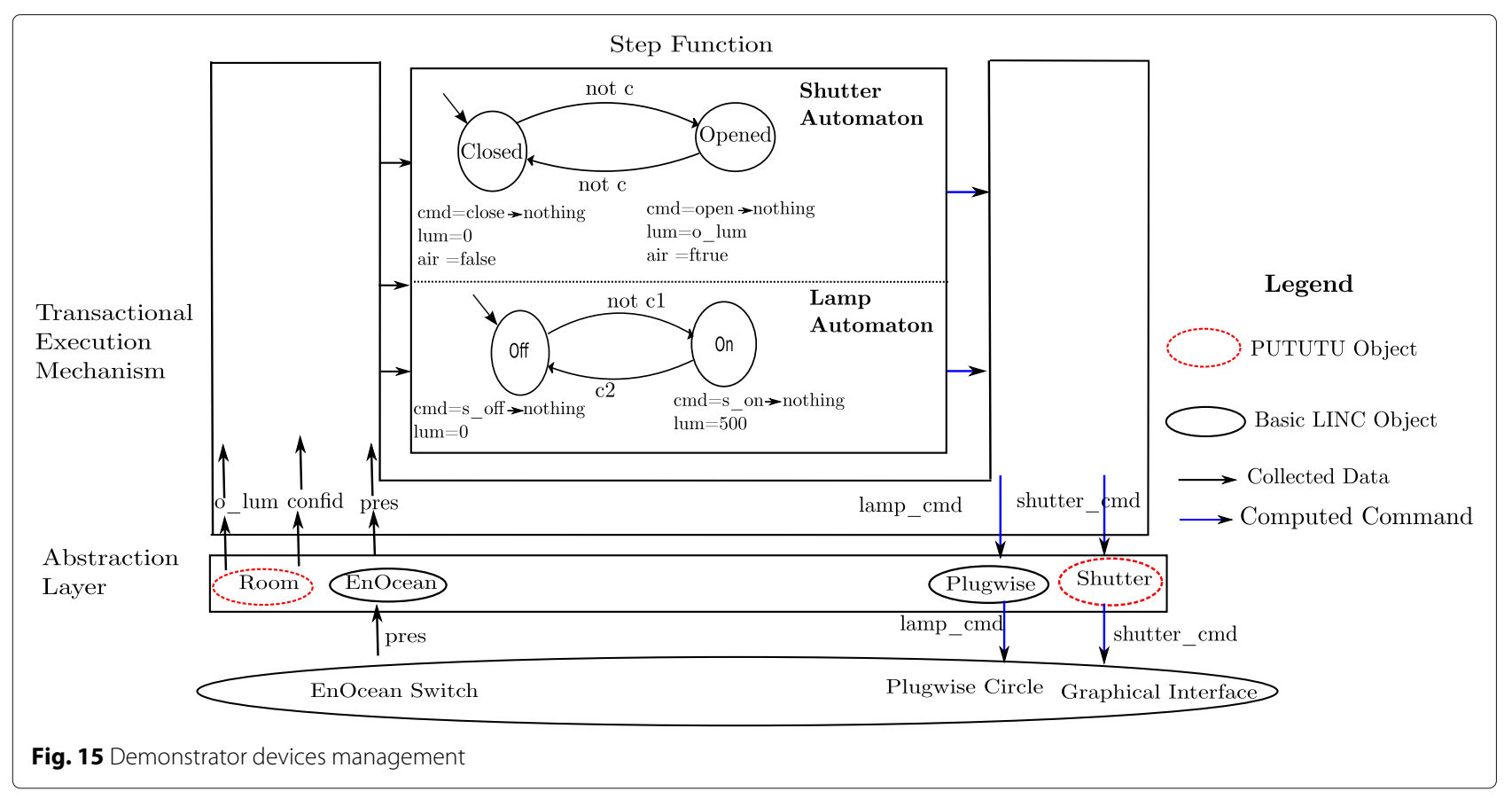

variables and the uncontrollable variables. The Table 2 shows the synthesis time of a controller for different rooms with different number of devices that do not have the same behaviour. The devices of each room are described using automata associated to variables. The Table shows that the synthesis time grows exponentially with the number of variables of the model.

When the number of variables is high, the synthesis of the controller can take a lot of time or not succeed to due limitations of CPU and/or RAM. In our approach, the generic environment model allows to deal with this limitation. All the devices that have the same behaviour are seen as one device and hence they are modelled using one single automaton and the associated variables. This improves the scalability by reducing the controller synthesis time. However, this is limiting for a high number of devices that have different behaviours, several automata must be used. In this case, the devices are managed by designing several autonomic loops, as explained in the Section 5.4.3.

Table 2 Design costs comparison

\begin{tabular}{ll}
\hline Considered rooms & Synthesis time \\
\hline R1 (6 devices/65 variables) & $1.4 \mathrm{~s}$ \\
R2 (12 devices/101 variables) & $31 \mathrm{~s}$ \\
R3 (18 devices/137 variables) & $797 \mathrm{~s}$ \\
R4 (24 devices/173 variables) & $4888 \mathrm{~s}$ \\
R9 (48 devices/353variables) & $10920 \mathrm{~s}$ \\
\hline
\end{tabular}

\subsubsection{Runtime cost evaluation}

At runtime, the generated execution rule reads data from all sensors and other sources and invokes the step function to compute the commands. The step is similar to a set of if then else and has a runtime cost that is low. Hence, the runtime cost of the execution rule depends on the data it reads in its precondition part.

The fact that the execution rule reads data from all sensors and other sources leads to a runtime cost that is not negligible, when the number of devices is high. The reason is that the middleware LINC is used to design reactive rules. To not miss an event and to react as soon as it occurs, a rule is executed by building an inference tree from the data read in its precondition. Hence, the more a rule reads data, the bigger is the inference tree. This slows down the rule execution.

However, to avoid conflicts and ensure reliability, it is necessary to read data from all sensors and have a global view of the environment. For instance, opening a window to cool a room requires not only data from temperature sensors but also outdoor noise, $\mathrm{CO}_{2}$ and pollen sensors to not violate other target objectives.

\subsubsection{Design cost and runtime cost improvement}

When the number of devices is high, they are managed as follows. The devices are first divided into several sets. Then, a loop, as the one presented in Fig. 1, is designed for each set of devices. These loops can be combined in different modes (i.e., parallel, coordinated, hierarchic) depending on the interactions between the devices sets 
(e.g., independent) and the structure of the considered smart environment (e.g., a building is composed of floors that consist of rooms).

Managing the devices by designing multiple loops improves the scalability of our approach, by reducing the design cost. Indeed, the controller synthesis is done for each devices set (not in all the devices) and can be performed modularly [34]. This also improves the specification by allowing developers, when providing the environment model, to consider sets of limited devices instead of all the devices. Finally, this allows for the distribution of the loops and improves the execution cost. More details on how smart environment devices are managed using multiple loops can be found in [37].

\subsection{Discussion about the case study and qualitative comparison with related work approaches}

The proposed framework allows developers to generate an executable model for the management of smart environment devices. To enable the executable model generation, developers provide a model of the considered environment and the target objective. The environment model is defined by specifying for each device, its states, its state transitions and its effects on the environment parameters. The objectives are defined by specifying the values the environment parameters must take. From the environment model and the objectives are generated a controller and an execution rule. At runtime, the execution rule collects data from the environment and invokes the controller that makes appropriate decisions to reliably achieve the objectives. In the followings, our approach is compared with the related work approaches to show its advantages.

\subsubsection{Comparison with rule based approaches}

In rule based approaches (e.g., $[5,6])$, developers define a set of rules to specify the actions to perform when events occur. These approaches are intuitive for the management of smart environment devices [38]. However, developers have to manually consider all the possible cases to define a set of complete rules. This prevents the system from being in a state where no action can be performed because the corresponding rules are not defined. For a large system, manually considering all the possible cases is tedious.

In our approach, all the possible cases are computed through discrete controller synthesis and a controller that decides the actions to perform depending on the events that occur is generated. This allows developers to not manually consider all the cases and this ensures that there is no conflicts and violations of objectives.

\subsubsection{Comparison with model checking based approaches}

In model checking based approaches (e.g., [3, 4]), developers first model the entities of the considered system and how they interact to achieve the target objectives (i.e., decide the actions to perform when events occur). Then, developers define a set of properties and the model checker verify if the properties are satisfied by the given model. If a property is not satisfied, the model is modified and verified again. When the properties are satisfied, developers implement the corresponding executable model, for instance by generating rules from the verified model, as done in [13].

Verifying the model prevents from conflicts and objectives violations. This ensures the reliability of the smart environment. The model verification relies on the state space exploration techniques and is, similarly to our approach, exponential in the number of variables of the model. The execution of the executable model is also the same in both approaches. The advantages of our approach is that developers do not have to model how the devices interact to achieve the objectives (i.e., decision making) and do not need to modify the model when a property is not satisfied. Developers just describe each devices (states, transitions, effects on the environment) and the objectives. The decisions are reliably made by a controller that is generated.

\section{Related work}

In $[2-4,13,14,39]$, the authors propose model checking based approaches for reliable smart environments. These approaches consist in first modelling the desired behaviour of the considered smart environment (i.e., the behaviour of the devices and how they interact to achieve the target objectives). Then, verifying if a set of properties, expressed in temporal logics, are valid in the designed model, using a model checker. In the opposite, our approach does not require to model the desired behaviour or verify properties. The properties are, automatically, enforced on a model that describes the features of each device (i.e., effects on the environment, states and transitions). For this reason, our approach is more convenient than those based on model checking. It allows to declaratively obtain the desired behaviour of the considered smart environment. The advantage of model checking based approaches is that several methods have been proposed for the reduction of the verification cost. To benefit from these methods, for a large system, one can combine model checking with our approach. In this case, the considered system is first divided into several subsystems. Then, the subsystems with a desired behaviour that can (resp. cannot) be easily specified manually are designed using a model checking based approach (resp. our approach).

In $[1,5-9,16]$, the authors propose approaches for reliable rule based smart environments. They first consider a set of errors that can occur within a set of 
rules (e.g., conflicts, circularities, constraints violations, redundancy). Then, they propose methods to detect (e.g., pairwise comparison, model checking) and solve (e.g., priority) the considered errors. These approaches do not enable the detection of implicit errors. Indeed, they do not consider the effects of the rules on the environment. Our approach prevents from both implicit conflicts and constraint violations, by considering the devices effects on the environment.

In $[11,12,40]$, the authors propose environment aware approaches for the reliable smart environments. These approaches enable the detection of implicit conflicts and/or objectives violations, by considering the effects of devices. However, using these approaches require to manually solve the detected conflicts and objectives violations. In addition, they do not consider the inconsistencies that are due to communication errors and hardware failures, as done in our approach.

In $[32,41]$, the authors propose an approach that handle inconsistencies. This approach consists in verifying if the actual effect of an action, on an actuator, is equal to its expected effect, using data from sensors or other sources. An inconsistency is assumed if the actual effect of an action is different from its expected effect. In this case, the action is performed again using an alternative actuator. The limitation of this approach is that it can take a lot of time to detect an inconsistency, for instance when the effect of the action is not instantaneous (e.g., temperature variation). In our approach, when performing an action, the fact that the actuator is faulty or unreachable due to a communication error is detected. This prevents from taking a lot of time to detect that the action was not performed. However, an actuator can become faulty after the action is done. Such a failure is not detected by our approach. A possible solution to detect such a failure is to verify the actual effects of actions, using data from sensors. For this reason, the approach proposed in $[32,41]$ is complementary to our approach.

In [42], the authors propose an approach that prevents from implicit conflicts among a set of ECA rules and enables the detection inconsistencies. For this, each rule specifies its post-condition (expected effect). Postconditions are used to detect conflicts and inconsistencies. A conflict is detected at design time when the same event triggers rules with contradictory post-conditions or at runtime when such rules are triggered by different events. Conflicts detected at compilation time are solved by users and those detected at runtime are solved by a set of resolution rules. An inconsistency occurs if the actual effect of a rule is different from its post-condition. In this case, a failure event is raised to trigger alternative rules (if they are defined). This approach is limited because users have to solve conflicts and also it takes time to detect an inconsistency, when the effect of the involved rule is not instantaneous.

In [43], the authors propose an approach that enables the detection of errors (conflicts and objectives violations) among a set of rules. This approach also enables the detection of inconsistencies by verifying the actions actual effects. In a more recent work [44], the authors consider environment dependencies for the detection of implicit errors. However, using this approach requires to solve the detected errors. Moreover, the fact that an actuator is faulty is not detected when performing an action on it, as done in our approach, but after a certain time when the expected effect is not observed.

\section{Conclusion}

This paper has proposed a framework for reliable and environment aware management of smart environment devices. This framework enables the declarative management of devices, hides their heterogeneity, prevents from inconsistencies (due to communication errors or actuators failure), and conflicting decisions including those caused by environment dependencies. This is done following the autonomic computing principles. First, an abstraction layer is used to collect data from the environment. Then, based on the data and knowledge about the environment, a generated controller computes appropriate commands that allow to reach the target objectives without conflict. Finally, a transactional execution mechanism is used to atomically send the computed commands to the actuators and update the knowledge to prevent from inconsistencies.

In current status, the proposed framework has two main limitations. First, developers have to describe the behaviours of actuators and specify their effects on the environment. Hence, the reliability of the devices management depends on the accuracy of the descriptions given by developers. Second, when performing an action on a actuator that is not a soft actuator, if the action cannot be performed (e.g., due to a communication error), a SMS is sent to the maintenance team and nothing else is done meaning that the controller stops until the problem is solved by the maintenance.

An important perspective of this work is to improve the handling of communication errors and actuators failures, by performing alternative actions before informing the maintenance. The other perspective is to provide a domain specific language that enables home occupants or building managers to specify their devices (i.e., sensors, actuators) and the objectives they want to achieve. Then, the goal will be to generate from these specifications an executable model that automatically and reliably manages their homes or buildings devices. This executable model can consist of the composition of multiple loops, as illustrated in [37]. 


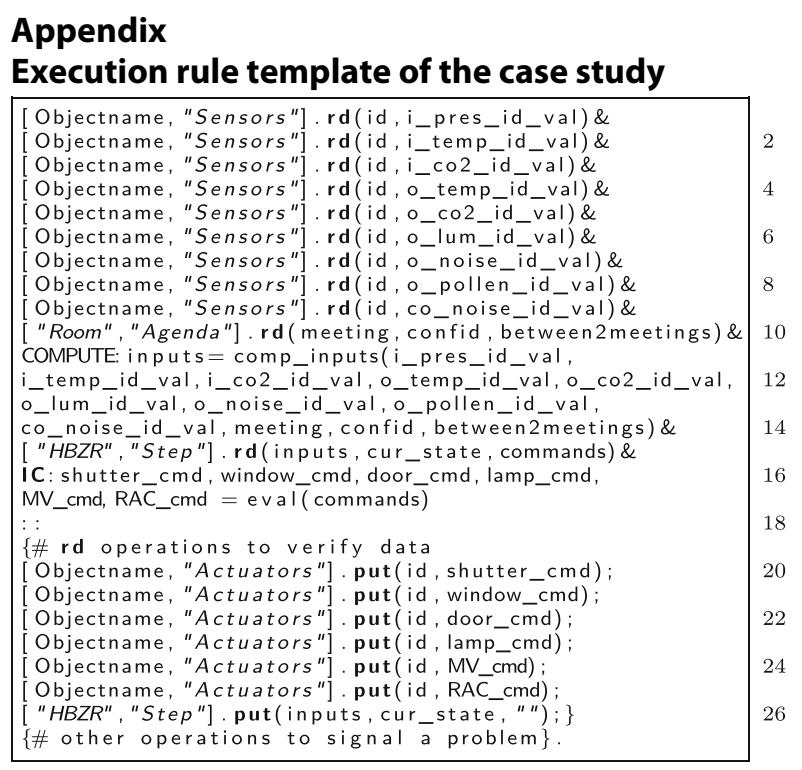

Listing 9 Generated room Execution rule template

\section{Abbreviations}

DCS: Discrete controller synthesis; MV: Mechanical ventilation; RAC: Reversible air conditioner

\section{Funding}

This work is funded by the H2020 TOPAs project (grant 676760).

\section{Authors' contributions}

This work is done in the context of the Ph.D. studies of ANS under the supervision of $M L$ and $E R$. All authors read and approved the final manuscript.

\section{Ethics approval and consent to participate}

Not applicable.

\section{Consent for publication}

Not applicable.

\section{Competing interests}

The authors declare that they have no competing interests.

\section{Publisher's Note}

Springer Nature remains neutral with regard to jurisdictional claims in published maps and institutional affiliations.

\section{Author details}

1 Université Grenoble Alpes, CEA, LETI, DACLE, LIALP, F-38000 Grenoble, France. ${ }^{2}$ Université Grenoble Alpes, INRIA, CNRS, Grenoble INP, LIG, F-38000 Grenoble, France.

Received: 15 February 2017 Accepted: 31 October 2017

Published online: 21 November 2017

\section{References}

1. Cano J, Delaval G, Rutten E. Coordination of ECA rules by verification and control. In: International Conference on Coordination Languages and Models. Berlin: Springer; 2014. p. 33-48.

2. Bonhomme S, Campo E, Esteve D, Guennec J. Methodology and tools for the design and verification of a smart management system for home comfort. In: Intelligent Systems, 2008. IS'08. 4th International IEEE Conference. vol. 3. Varna: IEEE; 2008. p. 24-2.

3. Corno F, Sanaullah M. Modeling and formal verification of smart environments. Secur Commun Netw. 2014;7(10):1582-98.

4. Augusto JC, Hornos MJ. Software simulation and verification to increase the reliability of intelligent environments. Adv Eng Softw. 2013;58:18-34.
5. Nacci AA, Balaji B, Spoletini P, Gupta R, Sciuto D, Agarwal Y. Buildingrules: a trigger-action based system to manage complex commercial buildings. In: Adjunct Proceedings of the 2015 ACM International Joint Conference on Pervasive and Ubiquitous Computing and Proceedings of the 2015 ACM International Symposium on Wearable Computers. New York: ACM; 2015. p. 381-384.

6. Sun Y, Wang X, Luo H, Li X. Conflict detection scheme based on formal rule model for smart building systems. IEEE Trans Human-Machine Syst. 2015;45(2):215-27.

7. Magill E, Blum J. Exploring conflicts in rule-based sensor networks. Pervasive Mob Comput. 2016;27:133-54.

8. Vannucchi C, Diamanti M, Mazzante G, et al. Symbolic verification of event-condition-action rules in intelligent environments. J Reliab Intell Environ. 2017;3(2):1-14

9. Le Guilly T, Nielsen MK, Pedersen T, et al. User constraints for reliable user-defined smart home scenarios. J Reliab Intell Environ. 2016;2(2): 75-91.

10. Le Guilly T, Smedegård JH, Pedersen T, Skou A. To do and not to do: constrained scenarios for safe smart house. In: Intelligent Environments (IE), 2015 International Conference on. Prague: IEEE; 2015. p. 17-24.

11. Maternaghan C, Turner KJ. Policy conflicts in home automation. Comput Netw. 2013;57(12):2429-41.

12. Liang CM, Karlsson BF, et al. SIFT: building an internet of safe things. In: Proceedings of the 14th International Conference on Information Processing in Sensor Networks. New York: ACM; 2015. p. 298-309.

13. Sylla AN, Louvel M, Pacull F. Coordination Rules Generation from Coloured Petri Net Models. In: Proceedings of the Int. Workshop on Petri Nets and Software Engineering (PNSE'15). Brussels: Daniel Moldt and al.; 2015. p. 325-326.

14. Augusto JC, Hornos MJ. Using Simulation and Verification to Inform the Development of Intelligent Environments. In: Intelligent Environments (Workshops). Guanajuato: IOS Press; 2012. p. 413-424.

15. Corno F, Sanaullah M. Formal verification of device state chart models. In: Intelligent Environments (IE), 2011 7th International Conference on. Nottingham: IEEE; 2011. p. 66-73.

16. Shehata M, Eberlein An, Fapojuwo A. Using semi-formal methods for detecting interactions among smart homes policies. Sci Comput Program. 2007;67(2-3):125-61.

17. Louvel M, Pacull F. Linc: A compact yet powerful coordination environment. In: Coordination Models and Languages. Berlin: Springer; 2014. p. 83-98.

18. Delaval G, Rutten É, Marchand H. Integrating discrete controller synthesis into a reactive programming language compiler. Discret Event Dyn Syst. 2013;23(4):385-418.

19. Kephart JO, Chess DM. The vision of autonomic computing. Computer. 2003;36(1):41-50

20. Pacull F, Ducreux LF, Thior $S$, et al. Self-organisation for building automation systems: Middleware linc as an integration tool. In: Industrial Electronics Society, IECON 2013-39th Annual Conference of the IEEE. Vienna: IEEE; 2013. p. 7726-7732.

21. Ducreux LF, Guyon-Gardeux C, et al. Resource-based middleware in the context of heterogeneous building automation systems. In: IECON 2012-38th Annual Conference on IEEE Industrial Electronics Society. Montreal: IEEE; 2012. p. 4847-4852.

22. Ducreux LF, Louvel M, et al. Dynamic Reconfiguration of Building Automation Systems with LINC. Sensors Transducers. 2015;185(2):68.

23. Iris H, Pacull F. Smart sensors and actuators: A question of discipline. Sensors Transducers. 2013;18(1):14.

24. Vergara-Gallego MI, Mokrenko O, et al. Implementation of an Energy Management Control Strategy for WSNs using the LINC Middleware. In: Proceedings of the 2016 International Conference on, Embedded Wireless Systems and Networks. Graz: Junction Publishing; 2016. p. 53-58.

25. Louvel M, Pacull F, Vergara-Gallego MI. Reliable control through wireless networks. In: Industrial Electronics Society, IECON 2016-42nd Annual Conference of the IEEE. IEEE; 2016. p. 4922-4927.

26. Carriero N, Gelernter D. Linda in context. Commun ACM. 1989;32(4): 444-58

27. Cooper T. Rule-based programming under OPS5, vol. 988. USA: Morgan Kaufmann Publishers Inc.; 1988.

28. Bernstein PA, Hadzilacos V, Goodman N. Concurrency control and recovery in database systems. vol. 370. Addison-wesley New York; 1987. 
29. Sylla AN, Louvel M, Rutten É. Combining Transactional and Behavioural Reliability in Adaptive Middleware. In: Proceedings of the 15th International Workshop on Adaptive and Reflective Middleware. New York: ACM; 2016. p. 5.

30. Bourcier J, Diaconescu A, Lalanda P, McCann JA. Autohome: An autonomic management framework for pervasive home applications. ACM Trans Auton Adapt Syst. 2011;6(1):8.

31. Cetina C, Giner P, Fons J, Pelechano V. Autonomic computing through reuse of variability models at runtime: The case of smart homes. Computer. 2009;42(10):

32. Seiger R, Huber S, Heisig P, Assmann U. Enabling Self-adaptive Workflows for Cyber-physical Systems. In: International Workshop on Business Process Modeling, Development and Support. Ljubljana: Springer; 2016. p. 3-17.

33. Warriach EU, Ozcelebi T, Lukkien JJ. Self-* Properties in Smart Environments: Requirements and Performance Metrics. In: Intelligent Environments (Workshops). Shanghai: IOS Press; 2014. p. 194-205.

34. Delaval G, Gueye SM, et al. Modular coordination of multiple autonomic managers. In: Proceedings of the 17th int. ACM Sigsoft symposium on Component-based software engineering. New York: ACM; 2014. p. 3-12.

35. Plugwise. Plugwise Circle. https://www.plugwise.com/circle/. Accessed 10 Nov 2017.

36. EnOcean. EnOcean Switch. https://www.enocean.com/en/ enocean_modules/ptm-210/. Accessed 10 Nov 2017.

37. Sylla AN, Louvel M, Rutten E, Delaval G. Design Framework for Reliable Multiple Autonomic Loops in Smart Environments. In: 2017 IEEE International Conference on, Cloud and Autonomic Computing (ICCAC). Tucson, AZ, United States; 2017. To appear. Available from:https://hal-cea. archives-ouvertes.fr/cea-01570026. Accessed 10 Nov 2017.

38. Ur B, McManus E, Pak Yong Ho M, Littman ML. Practical trigger-action programming in the smart home. In: Proceedings of the SIGCHI Conference on Human Factors in Computing Systems. New York: ACM; 2014. p. 803-812.

39. Pedersen T, Le Guilly T, et al. A method for model checking feature interactions. In: Software Technologies (ICSOFT) 2015 10th International, Joint Conference on. vol. 1. Colmar: IEEE; 2015. p. 1-10.

40. Nakamura M, Ikegami K, Matsumoto S. Considering impacts and requirements for better understanding of environment interactions in home network services. Comput Netw. 2013;57(12):2442-53.

41. Seiger R, Huber S, Schlegel T. Toward an execution system for self-healing workflows in cyber-physical systems. Softw Syst Model. 2016;1-22.

42. Shankar CS, Ranganathan A, Campbell R. An ECA-P policy-based framework for managing ubiquitous computing environments. In: Mobile and Ubiquitous Systems: Networking and Services, 2005. The Second Annual International Conference on. San Diego: IEEE; 2005. p. 33-42.

43. Preuveneers $D$, Berbers $Y$. Consistency in context-aware behavior: a model checking approach. In: Workshop Proceedings of the 8th International Conference on Intelligent Environments. vol. 13. Guanajuato: IOS Press; 2012. p. 401-412.

44. Preuveneers $D$, Joosen W. Semantic analysis and verification of context-driven adaptive applications in intelligent environments. J Reliab Intell Environ. 2016;2(2):53-73.

\section{Submit your manuscript to a SpringerOpen ${ }^{\circ}$ journal and benefit from:}

- Convenient online submission

- Rigorous peer review

- Open access: articles freely available online

- High visibility within the field

- Retaining the copyright to your article 